\title{
Enantioselective C-C Bond Cleavage Creating of Chiral Quaternary Carbon Centers
}

Takanori Matsuda, Masanori Shigeno, Masaomi Makino, and Masahiro Murakami*

Department of Synthetic Chemistry and Biological Chemistry, Kyoto University, Katsura, Kyoto 615-6510, Japan

\section{Supporting Information}

General. All reactions were carried out with standard Schlenk techniques under an argon atmosphere. Preparative thin-layer chromatography was performed with silica gel $60 \mathrm{PF}_{254}$ (Merck). ${ }^{1} \mathrm{H}$ and ${ }^{13} \mathrm{C}$ NMR spectra were recorded on a Varian Gemini $2000\left({ }^{1} \mathrm{H}\right.$ at 300.07

$\mathrm{Hz}$ and ${ }^{13} \mathrm{C}$ at $75.46 \mathrm{~Hz}$ ) spectrometer. ${ }^{11} \mathrm{~B}$ NMR spectra were recorded on a Varian Mercury $400\left({ }^{11} \mathrm{~B}\right.$ at $\left.128.48 \mathrm{~Hz}\right)$. All NMR data were obtained in $\mathrm{CDCl}_{3}$. Proton chemical shifts were referenced to the residual proton signal of the solvent at $7.26 \mathrm{ppm}$. Carbon chemical shifts were referenced to the carbon signal of the solvent at $77.00 \mathrm{ppm}$. Boron chemical shifts were referenced to an external standard $\mathrm{BF}_{3} \cdot \mathrm{OEt}_{2}$. High resolution mass spectra were recorded on a JEOL JMS-SX102A spectrometer. Infrared spectra were recorded on a Shimadzu FTIR-8100 spectrometer.

Materials. 1,4-Dioxane was distilled from sodium-benzophenone ketyl. Dimethyltitanocene was prepared according to the literature method. ${ }^{1}(S)$-SEGPHOS was supplied from Takasago International Corporation. All other commercially available chemical resources were used as received.

\section{Preparation of Boryl-Substituted Cyclobutanones 3a-c and 8}

(1) Ndakala, A. J.; Hashemzadeh, M.; So, R. C.; Howell, A. R. Org. Lett. 2002, 4, 1719. 

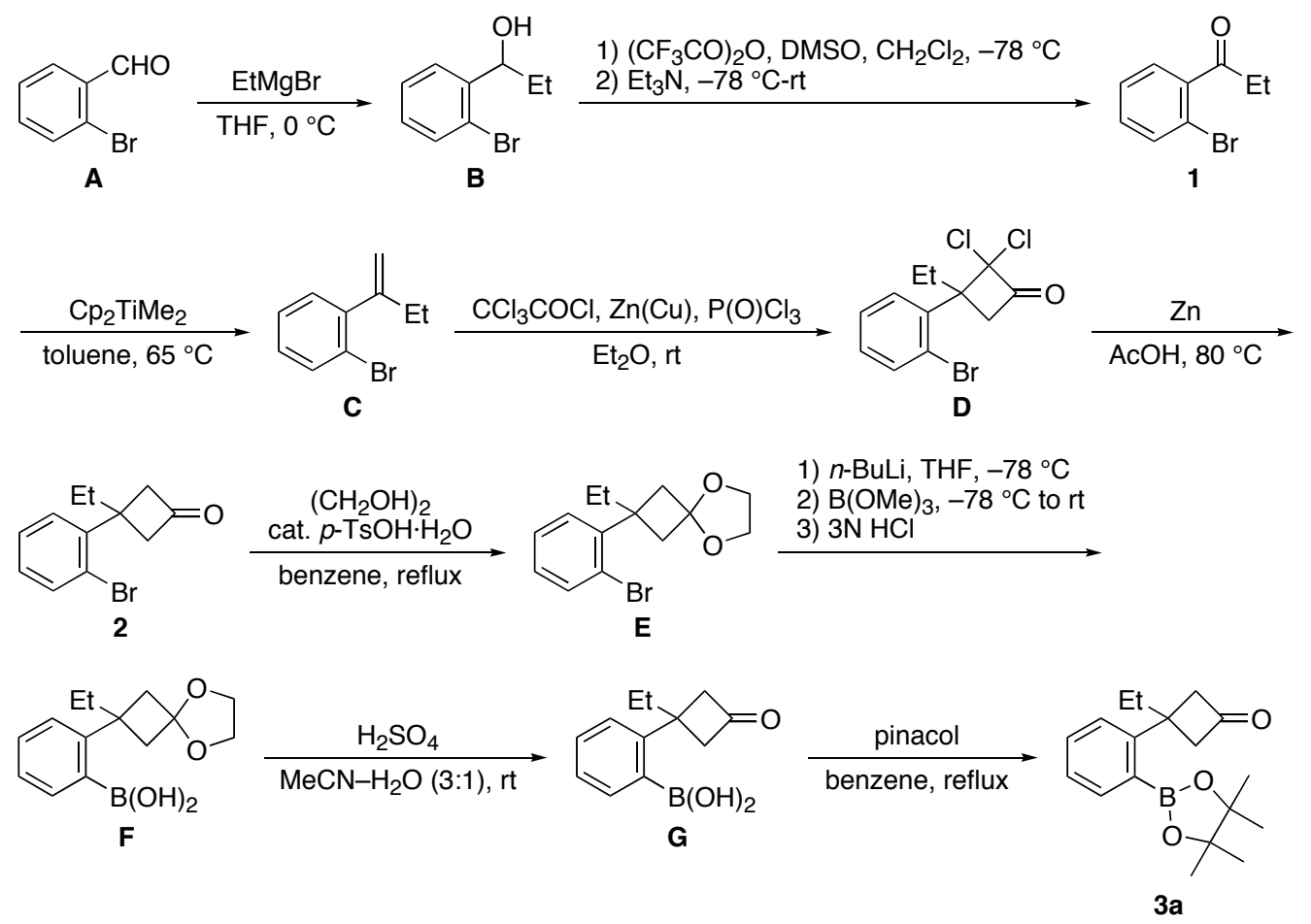

1-(2-Bromophenyl)propan-1-one (1). ${ }^{2}$ To a solution of 2-bromobenzaldehyde (A, $11.1 \mathrm{~g}$, $60.0 \mathrm{mmol})$ in THF $(100 \mathrm{~mL})$ was added dropwise EtMgBr in THF (1.0 M, $100 \mathrm{~mL}, 100$ mmol) at $0{ }^{\circ} \mathrm{C}$. After stirring at room temperature overnight, the reaction mixture was quenched with saturated $\mathrm{NH}_{4} \mathrm{Cl}$ aqueous solution and extracted with AcOEt. The extract was washed with brine, dried over $\mathrm{Na}_{2} \mathrm{SO}_{4}$, and concentrated. The residue was purified by column chromatography on silica gel (hexane:AcOEt $=4: 1)$ to afford alcohol $\mathbf{B}(8.53 \mathrm{~g}$, $39.7 \mathrm{mmol}, 66 \%)$.

To a solution of DMSO $(1.04 \mathrm{~g}, 13.3 \mathrm{mmol})$ in $\mathrm{CH}_{2} \mathrm{Cl}_{2}(10 \mathrm{~mL})$ were added dropwise a solution of trifluoroacetic anhydride $(2.09 \mathrm{~g}, 10.0 \mathrm{mmol})$ in $\mathrm{CH}_{2} \mathrm{Cl}_{2}(5 \mathrm{~mL})$ at $-78{ }^{\circ} \mathrm{C}$. After stirring for $10 \mathrm{~min}$, a solution of $\mathbf{B}(1.43 \mathrm{~g}, 6.63 \mathrm{mmol})$ in $\mathrm{CH}_{2} \mathrm{Cl}_{2}(10 \mathrm{~mL})$ was added dropwise to the mixture. The reaction mixture was allowed to room temperature, and $\mathrm{Et}_{3} \mathrm{~N}$ $(1.81 \mathrm{~g}, 17.9 \mathrm{mmol})$ was added by syringe. After stirring at room temperature for $1 \mathrm{~h}$, water was added to the reaction mixture, and the mixture was extracted with $\mathrm{CH}_{2} \mathrm{Cl}_{2}$ three times. The combined organic layer was washed with $\mathrm{NaHCO}_{3}$ and brine, dried over $\mathrm{MgSO}_{4}$, and concentrated. The residue was purified by column chromatography on silica gel

(2) [62403-86-5] 
(hexane:AcOEt = 8:1) to afford $1(1.01 \mathrm{~g}, 4.74 \mathrm{mmol}, 71 \%):{ }^{1} \mathrm{H} \mathrm{NMR} \delta 1.20(\mathrm{t}, J=7.2 \mathrm{~Hz}$, $3 \mathrm{H}), 2.91(\mathrm{t}, J=7.2 \mathrm{~Hz}, 2 \mathrm{H}), 7.23-7.31(\mathrm{~m}, 1 \mathrm{H}), 7.31-7.36(\mathrm{~m}, 2 \mathrm{H}), 7.58(\mathrm{dd}, J=8.6,0.8$ $\mathrm{Hz}, 1 \mathrm{H})$.

3-(2-Bromophenyl)-3-ethylcyclobutanone (2). To a solution of $\mathbf{1}$ (739 $\mathrm{mg}, 3.47 \mathrm{mmol}$ ) in toluene $(20 \mathrm{~mL})$ was added dropwise a solution of $\mathrm{Cp}_{2} \mathrm{TiMe}_{2}(1.44 \mathrm{~g}, 6.9 \mathrm{mmol})$ in toluene $(20 \mathrm{~mL})$. After stirring at $65^{\circ} \mathrm{C}$ for $24 \mathrm{~h}$ in the dark, the mixture was poured into hexane. The resulting orange solid was filtered off, and the filtrate was evaporated. The residue was purified by column chromatography on silica gel (hexane) to give olefin $\mathbf{C}$.

To a mixture of zinc-copper couple (411 mg, $6.28 \mathrm{mmol}), \mathbf{C}, \mathrm{Et}_{2} \mathrm{O}(10 \mathrm{~mL})$ was added dropwise a solution of $\mathrm{CCl}_{3} \mathrm{COCl}(947 \mathrm{mg}, 5.21 \mathrm{mmol})$ and $\mathrm{P}(\mathrm{O}) \mathrm{Cl}_{3}(799 \mathrm{mg}, 5.21 \mathrm{mmol})$ in $\mathrm{Et}_{2} \mathrm{O}(5 \mathrm{~mL})$ over $15 \mathrm{~min}$. After stirring at room temperature for $7 \mathrm{~h}$, the reaction mixture was filtered $\left(\right.$ Celite $\left.^{\circledR}, \mathrm{Et}_{2} \mathrm{O}\right)$. The filtrate was washed with saturated $\mathrm{NaHCO}_{3}$ aqueous solution and brine, dried over $\mathrm{MgSO}_{4}$, and concentrated to afford dichlorocyclobutanone $\mathbf{D}$. To a suspension of zinc $(1.13 \mathrm{~g}, 17.3 \mathrm{mmol})$ in $\mathrm{AcOH}(5 \mathrm{~mL})$ was added dropwise the crude $\mathbf{D}$ in $\mathrm{AcOH}(5 \mathrm{~mL})$, and the reaction mixture was heated at $80{ }^{\circ} \mathrm{C}$ for $2 \mathrm{~h}$. The reaction mixture was filtered through a pad of Celite ${ }^{\circledR}$ and washed with $\mathrm{Et}_{2} \mathrm{O}$. The ethereal solution was washed with water, saturated $\mathrm{NaHCO}_{3}$ aqueous solution, and brine. The organic phase was dried over $\mathrm{MgSO}_{4}$ and concentrated. The residue was purified by column chromatography on silica gel (hexane:AcOEt = 8:1) to afford cyclobutanone $2(651$ $\mathrm{mg}, 2.57 \mathrm{mmol}, 74 \%):{ }^{1} \mathrm{H} \mathrm{NMR} \delta 0.71(\mathrm{t}, J=7.35 \mathrm{~Hz}, 3 \mathrm{H}), 2.00$ (br s, 2H), 3.17-3.28 (m, 2H), 3.41-3.50 (m, 2H), 7.08-7.14 (m, 1H), 7.16-7.20 (m, 1H), 7.26-7.32 (m, 1H), $7.58(\mathrm{dd}$, $J=8.0,1.4 \mathrm{~Hz}, 1 \mathrm{H})$.

\section{3-Ethyl-3-[2-(4,4,5,5-tetramethyl-1,3,2-dioxaborolan-2-yl)phenyl]cyclobutanone (3a).}

A solution of $2(651 \mathrm{mg}, 2.57 \mathrm{mmol}), p-\mathrm{TsOH} \cdot \mathrm{H}_{2} \mathrm{O}(24.7 \mathrm{mg}, 0.13 \mathrm{mmol})$, ethylene glycol $(1.0 \mathrm{~mL})$ in benzene $(15 \mathrm{~mL})$ was refluxed under a Dean-Stark trap for $3 \mathrm{~h}$. The mixture was cooled to room temperature, diluted with ether, and washed with saturated $\mathrm{NaHCO}_{3}$ aqueous solution and brine. The organic phase was dried over $\mathrm{MgSO}_{4}$ and concentrated. The residue was passed through a short column of silica gel (hexane:AcOEt $=8: 1$ ) to afford ethylene glycol ketal E (620 mg, 2.09 mmol, 81\%). 
To a stirred solution of $\mathbf{E}(620 \mathrm{mg}, 20.9 \mathrm{mmol})$ in $\mathrm{THF}(3 \mathrm{~mL})$ at $-78^{\circ} \mathrm{C}$ was added $n$-BuLi in hexane $(1.56 \mathrm{M}, 1.47 \mathrm{~mL}, 2.29 \mathrm{mmol})$. After stirring for $1 \mathrm{~h}$, a solution of trimethyl borate $(434 \mathrm{mg}, 4.17 \mathrm{mmol})$ in THF $(2 \mathrm{~mL})$ was added dropwise to the reaction mixture at $78^{\circ} \mathrm{C}$. The mixture was stirred at room temperature overnight, and $3 \mathrm{~N} \mathrm{HCl}(3 \mathrm{~mL})$ was added. The mixture was extracted with ether, washed with brine, dried over $\mathrm{Na}_{2} \mathrm{SO}_{4}$, and concentrated to give boronic acid $\mathbf{F}$.

The crude $\mathbf{F}$ was diluted with acetonitrile $(30 \mathrm{~mL})$ and $2 \mathrm{~N}$ sulfuric acid $(15 \mathrm{~mL})$, and the mixture was stirred at room temperature for $16 \mathrm{~h}$. The reaction was quenched by neutralization with aqueous $\mathrm{NaHCO}_{3}$, and the mixture was extracted with ether and washed with brine. The extract was dried over $\mathrm{Na}_{2} \mathrm{SO}_{4}$ and evaporated to afford cyclobutanone $\mathbf{G}$.

To the crude $\mathbf{G}$ was added pinacol (473 mg, $4.00 \mathrm{mmol})$ and benzene (10 mL), and the mixture was refluxed under a Dean-Stark trap for $3 \mathrm{~h}$. The mixture was cooled to room temperature, diluted with ether, and washed with saturated $\mathrm{NaHCO}_{3}$ aqueous solution and brine. The organic phase was dried over $\mathrm{Na}_{2} \mathrm{SO}_{4}$ and evaporated. The residue was purified by chromatography on silica gel (hexane:AcOEt = 8:1) to afford 3a (364 mg, $1.21 \mathrm{mmol}$, $58 \%):{ }^{1} \mathrm{H}$ NMR $\delta 0.74(\mathrm{t}, J=7.4 \mathrm{~Hz}, 1 \mathrm{H}), 1.32(\mathrm{~s}, 12 \mathrm{H}), 2.00(\mathrm{q}, J=7.4 \mathrm{~Hz}, 1 \mathrm{H}), 3.19-3.38$ $(\mathrm{m}, 4 \mathrm{H}), 7.15(\mathrm{~d}, J=7.8 \mathrm{~Hz}, 1 \mathrm{H}), 7.23(\mathrm{dt}, J=1.3,7.4 \mathrm{~Hz}, 1 \mathrm{H}), 7.38(\mathrm{dt}, J=1.6,7.6 \mathrm{~Hz}$, $1 \mathrm{H}), 7.79(\mathrm{dd}, J=7.4,1.7 \mathrm{~Hz}, 1 \mathrm{H}) ;{ }^{13} \mathrm{C} \mathrm{NMR} \delta 9.8,24.9,34.2,39.9,58.3,83.8,125.4$, $128.0,129.8,136.5,151.4,208.8$ [carbon attached to boron was not observed due to quadrupole broadening caused by the boron nucleus]; ${ }^{11} \mathrm{~B}$ NMR $\delta 30.4$; IR (neat) 2980, 1775, 1348, 1144, 912, $739 \mathrm{~cm}^{-1}$; HRMS (CI) calcd for $\mathrm{C}_{18} \mathrm{H}_{26} \mathrm{BO}_{3}\left(\mathrm{M}^{+}+\mathrm{H}\right) 301.1975$, found 301.1975. Anal. Calcd for $\mathrm{C}_{18} \mathrm{H}_{25} \mathrm{BO}_{3}$ : C, 72.02; H, 8.39. Found: C, 71.80; H, 8.31.

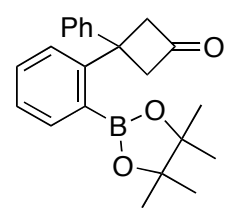

3-Phenyl-3-[2-(4,4,5,5-tetramethyl-1,3,2-dioxaborolan-2-yl)phenyl]cyclobutanone (3b). ${ }^{1} \mathrm{H}$ NMR $\delta 1.07(\mathrm{~s}, 12 \mathrm{H}), 3.67-3.89(\mathrm{~m}, 4 \mathrm{H}), 7.08-7.24(\mathrm{~m}, 5 \mathrm{H}), 7.27-7.34(\mathrm{~m}, 1 \mathrm{H}), 7.44-$ $7.52(\mathrm{~m}, 2 \mathrm{H}), 7.86(\mathrm{~d}, J=7.2 \mathrm{~Hz}, 1 \mathrm{H}) ;{ }^{13} \mathrm{C} \mathrm{NMR} \delta 24.6,42.9,62.0,83.6,125.8,126.0$, $126.3,127.5,128.0,130.5,137.1,148.5,151.2,208.2$ [carbon attached to boron was not 
observed due to quadrupole broadening caused by the boron nucleus]; ${ }^{11} \mathrm{~B}$ NMR $\delta 30.0$; HRMS (CI) calcd for $\mathrm{C}_{22} \mathrm{H}_{26} \mathrm{BO}_{3}\left(\mathrm{M}^{+}+\mathrm{H}\right) 349.1975$, found 349.1975 .<smiles>CC(C)(C)C(C)(C)OB1OC(=O)CC12CC(=O)CC21Pc2ccccc21</smiles>

\section{3-Isopropyl-3-[2-(4,4,5,5-tetramethyl-1,3,2-dioxaborolan-2-yl)phenyl]cyclobutanone}

(3c). ${ }^{1} \mathrm{H}$ NMR $\delta 0.87$ (d, $\left.J=7.2 \mathrm{~Hz}, 6 \mathrm{H}\right), 1.34$ (s, $12 \mathrm{H}$ ), 2.47 (sept, $J=6.8 \mathrm{~Hz}, 1 \mathrm{H}$ ), 3.20$3.32(\mathrm{~m}, 2 \mathrm{H}), 3.36-3.49(\mathrm{~m}, 2 \mathrm{H}), 7.17(\mathrm{~d}, J=7.8 \mathrm{~Hz}, 1 \mathrm{H}), 7.24(\mathrm{dt}, J=1.2,7.5 \mathrm{~Hz}, 1 \mathrm{H})$, $7.37(\mathrm{dt}, J=1.8,7.5 \mathrm{~Hz}, 1 \mathrm{H}), 7.80(\mathrm{dd}, J=7.2,1.5 \mathrm{~Hz}, 1 \mathrm{H}) ;{ }^{13} \mathrm{C}$ NMR $\delta 18.0,24.9,35.3$, $42.9,55.2,83.7,125.3,128.2,129.5,136.5,152.5,208.9$ [carbon attached to boron was not observed due to quadrupole broadening caused by the boron nucleus]; ${ }^{11} \mathrm{~B}$ NMR $\delta 31.0$; HRMS (FAB) calcd for $\mathrm{C}_{19} \mathrm{H}_{28} \mathrm{BO}_{3}\left(\mathrm{M}^{+}+\mathrm{H}\right) 315.2132$, found 315.2132 .

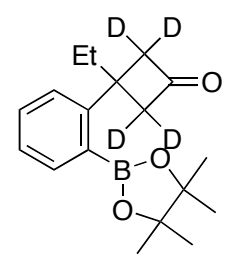

\section{3-Ethyl-3-[2-(4,4,5,5-tetramethyl-1,3,2-dioxaborolan-2-yl)phenyl]-[2,2,4,4-}

\section{$\left.{ }^{2} \mathbf{H}_{4}\right]$ cyclobutanone (3a- $\left.\boldsymbol{d}_{4}\right)$.}

A solution of 3a (308 mg, $1.03 \mathrm{mmol})$ and $\mathrm{K}_{3} \mathrm{PO}_{4}(109 \mathrm{mg}, 0.515 \mathrm{mmol})$ in 1,4-dioxane $(3.0 \mathrm{~mL})$ and $\mathrm{D}_{2} \mathrm{O}(0.6 \mathrm{ml})$ was heated at $100{ }^{\circ} \mathrm{C}$ for $12 \mathrm{~h}$. The reaction mixture was filtered (Florisi ${ }^{\circledR}$, AcOEt) and concentrated. A solution of the residue and $\mathrm{K}_{3} \mathrm{PO}_{4}(109 \mathrm{mg}, 0.515$ $\mathrm{mmol})$ in 1,4-dioxane $(3.0 \mathrm{~mL})$ and $\mathrm{D}_{2} \mathrm{O}(0.6 \mathrm{ml})$ was again heated, filtered, and concentrated. The residue was purified by preparative thin-layer chromatography (hexane:AcOEt = 8:1) to afford 3a- $d_{4}(272 \mathrm{mg}, 0.894 \mathrm{mmol}, 87 \%, 97 \% \mathrm{D})$.<smiles>Cc1ccc(Br)c(C(=O)CCCOc2ccccc2)c1</smiles> 
4-Benzyloxy-1-(2-bromo-5-methylphenyl)butan-1-one (7). ${ }^{1} \mathrm{H}$ NMR $\delta 2.04$ (quint, $J=$ $6.6 \mathrm{~Hz}, 2 \mathrm{H}), 2.30(\mathrm{~s}, 3 \mathrm{H}), 3.03(\mathrm{t}, J=6.9 \mathrm{~Hz}, 2 \mathrm{H}), 3.56(\mathrm{t}, J=6.0 \mathrm{~Hz}, 2 \mathrm{H}), 4.50(\mathrm{~s}, 2 \mathrm{H})$, 7.05-7.11 (m, 1H), $7.15(\mathrm{~d}, J=1.8 \mathrm{~Hz}, 1 \mathrm{H}), 7.25-7.35(\mathrm{~m}, 5 \mathrm{H}), 7.46(\mathrm{~d}, J=8.4 \mathrm{~Hz}, 1 \mathrm{H})$; ${ }^{13} \mathrm{C}$ NMR $\delta 20.8,24.2,39.4,69.2,72.9,115.1,127.5,127.6,128.3,129.0,132.2,133.3$, 137.4, 138.4, 141.6, 204.2; HRMS (FAB) calcd for $\mathrm{C}_{18} \mathrm{H}_{19} \mathrm{BrO}_{2}\left(\mathrm{M}^{+}+\mathrm{H}\right) 347.0647$, found 347.0647.

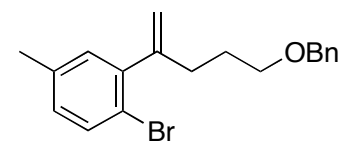

1-Bromo-4-methyl-2-(5-benzyloxypent-1-en-2-yl)benzene (7'). ${ }^{1} \mathrm{H}$ NMR $\delta$ 1.67-1.78 (m, 2H), 2.28 (s, 3H), $2.51(\mathrm{t}, J=7.8 \mathrm{~Hz}, 2 \mathrm{H}), 3.50(\mathrm{t}, J=6.6 \mathrm{~Hz}, 2 \mathrm{H}), 4.49(\mathrm{~s}, 2 \mathrm{H}), 4.93-4.96$ (m, 1H), 5.20-5.22 (m, 1H), 6.89-6.96 (m, 2H), 7.24-7.36 (m, 5H), $7.41(\mathrm{~d}, J=8.1 \mathrm{~Hz}, 1 \mathrm{H})$; ${ }^{13} \mathrm{C}$ NMR $\delta$ 20.8, 28.0, 33.4, 69.9, 72.9, 115.1, 118.5, 127.5, 127.6, 128.3, 129.2, 131.0, 132.4, 136.9, 138.5, 143.5, 149.4; HRMS (FAB) calcd for $\mathrm{C}_{19} \mathrm{H}_{22} \mathrm{BrO}\left(\mathrm{M}^{+}+\mathrm{H}\right) 345.0854$, found 345.0850 .

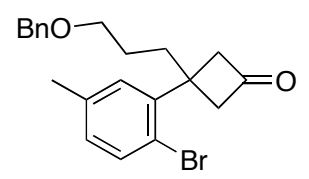

3-(3-Benzyloxypropyl)-3-(2-bromo-5-methylphenyl)cyclobutanone (7"). ${ }^{1} \mathrm{H}$ NMR $\delta$ 1.38 (quint, $J=7.8 \mathrm{~Hz}, 2 \mathrm{H}), 2.04(\mathrm{br} \mathrm{s}, 2 \mathrm{H}), 2.31(\mathrm{~s}, 3 \mathrm{H}), 3.19-3.30(\mathrm{~m}, 2 \mathrm{H}), 3.37(\mathrm{t}, J=$ $6.8 \mathrm{~Hz}, 2 \mathrm{H}), 3.43-3.52(\mathrm{~m}, 2 \mathrm{H}), 4.42(\mathrm{~s}, 2 \mathrm{H}), 6.93(\mathrm{dd}, J=8.1,2.1 \mathrm{~Hz}, 1 \mathrm{H}), 6.98(\mathrm{~d}, J=1.8$ $\mathrm{Hz}, 1 \mathrm{H}), 7.23-7.36(\mathrm{~m}, 5 \mathrm{H}), 7.44(\mathrm{~d}, J=8.1 \mathrm{~Hz}, 1 \mathrm{H}) ;{ }^{13} \mathrm{C}$ NMR $\delta 20.9,26.0,35.1,39.4$, 57.7, 69.9, 72.9, 118.9, 127.4, 127.5, 128.2, 129.1, 130.5, 134.0, 136.8, 138.1, 142.8, 206.2.

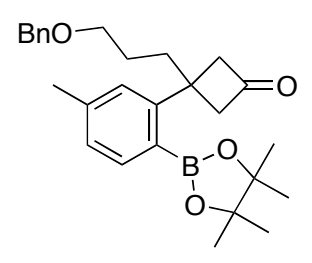




\section{3-(3-Benzyloxypropyl)-3-[5-methyl-2-(4,4,5,5-tetramethyl-1,3,2-dioxaborolan-2-yl)-}

phenyl]cyclobutanone (8). ${ }^{1} \mathrm{H}$ NMR $\delta 1.30$ (s, 6H), 1.31 (s, 6H), 1.39-1.53 (m, 2H), 2.02$2.11(\mathrm{~m}, 2 \mathrm{H}), 2.36(\mathrm{~s}, 3 \mathrm{H}), 3.21-3.42(\mathrm{~m}, 6 \mathrm{H}), 4.42(\mathrm{~s}, 2 \mathrm{H}), 6.97(\mathrm{~s}, 1 \mathrm{H}), 7.06(\mathrm{~d}, J=7.5 \mathrm{~Hz}$, $1 \mathrm{H}), 7.23-7.37(\mathrm{~m}, 5 \mathrm{H}), 7.73(\mathrm{dd}, J=7.5,0.9 \mathrm{~Hz}, 1 \mathrm{H}) ;{ }^{13} \mathrm{C}$ NMR $\delta 21.7,24.9,26.1,37.8$, $39.1,58.7,70.5,72.8,83.7,126.3,127.4,127.5,128.3,128.7,137.0,138.4,140.0,151.6$, 208.7 [carbon attached to boron was not observed due to quadrupole broadening caused by the boron nucleus]; ${ }^{11} \mathrm{~B}$ NMR $\delta$ 31.0; HRMS (FAB) calcd for $\mathrm{C}_{27} \mathrm{H}_{36} \mathrm{BO}_{4}\left(\mathrm{M}^{+}+\mathrm{H}\right)$ 435.2707, found 435.2704 .

\section{Rhodium-Catalyzed Asymmetric Addition/Ring-Opening of Boryl-Substituted Cyclobutanones}

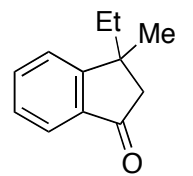

(S)-3-Ethyl-3-methylindan-1-one (4a). To a mixture of $\left[\mathrm{RhCl}\left(\mathrm{C}_{2} \mathrm{H}_{4}\right)_{2}\right]_{2}(2.72 \mathrm{mg}, 7.0 \mu \mathrm{mol}$, $3.5 \mathrm{~mol} \%$ ), (S)-SEGPHOS (8.58 mg, $14.1 \mu \mathrm{mol}, 7.0 \mathrm{~mol} \%$ ), and $\mathrm{K}_{3} \mathrm{PO}_{4}(21.2 \mathrm{mg}, 0.10$ $\mathrm{mmol})$ in water $(0.1 \mathrm{~mL})$ and 1,4-dioxane $(1.7 \mathrm{~mL})$ was added $3 \mathbf{a}(60.0 \mathrm{mg}, 0.20 \mathrm{mmol})$ in 1,4-dioxane $(0.30 \mathrm{~mL})$. After being stirred for $5 \mathrm{~h}$ at $100{ }^{\circ} \mathrm{C}$, the reaction mixture was filtered through a pad of Florisil ${ }^{\circledR}$ (ether-AcOEt). The filtrate was purified by preparative thin-layer chromatography of silica gel (hexane:AcOEt $=16: 1)$ to afford $4 a(33.4 \mathrm{mg}$, $0.192 \mathrm{mmol}, 96 \%):[\alpha]^{27}{ }_{\mathrm{D}}+7.4\left(c 0.53\right.$, benzene) $;{ }^{3}{ }^{1} \mathrm{H}$ NMR $\delta 0.72(\mathrm{t}, J=7.5 \mathrm{~Hz}, 3 \mathrm{H}), 1.39$ (s, 3H), 1.59-1.81 (m, 2H), $2.41(\mathrm{~d}, J=18.8 \mathrm{~Hz}, 1 \mathrm{H}), 2.64(\mathrm{~d}, J=18.8 \mathrm{~Hz}, 1 \mathrm{H}), 7.31-7.38$ (m, 1H), 7.40-7.45 (m, 1H), 7.55-7.62 (m, 1H), 7.66-7.70 (m, 1H); ${ }^{13} \mathrm{C}$ NMR $\delta 9.3,28.0$, 34.7, 42.4, 49.7, 123.3, 123.8, 127.4, 134.8, 136.1, 162.6, 206.1; IR (neat) 1786, 1717, 1605, 1462, 1287, 1237, $764 \mathrm{~cm}^{-1}$; HRMS (EI) calcd for $\mathrm{C}_{12} \mathrm{H}_{14} \mathrm{O}\left(\mathrm{M}^{+}\right)$174.1045, found 174.1045 .

(3) $(R)$-isomer: $[\alpha]_{D}^{22}-10.1$ (c 3.4, benzene). Hill, R. K.; Newkome, G. R. Tetrahedron 1969, 25, 1249. 
The enantiomeric excess was determined to be $95 \%$ by chiral GC (Chiraldex G-BP column, $100{ }^{\circ} \mathrm{C}(5 \mathrm{~min})$ then $5{ }^{\circ} \mathrm{C} / \mathrm{min}$ to $120{ }^{\circ} \mathrm{C}(22 \mathrm{~min})$; retention times: $t_{1}=15.2 \mathrm{~min}(S), t_{2}=$ $16.8 \min (R))$.

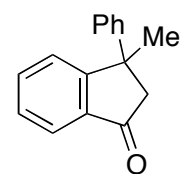

3-Methyl-3-phenylindan-1-one (4b). ${ }^{4}$ According to the procedure analogous to that described for 4a, 4b (41.4 mg, $0.186 \mathrm{mmol}, 93 \%)$ was prepared from $\mathbf{3 b}$ (69.6 mg, 0.20 mmol). 4b: $[\alpha]_{\mathrm{D}}^{25}-23.6\left(c 0.33, \mathrm{CHCl}_{3}\right) ;{ }^{1} \mathrm{H} \mathrm{NMR} \delta 1.83(\mathrm{~s}, 3 \mathrm{H}), 2.86(\mathrm{~d}, J=19.1 \mathrm{~Hz}, 1 \mathrm{H})$, $2.99(\mathrm{~d}, J=19.1 \mathrm{~Hz}, 1 \mathrm{H}), 7.15-7.22(\mathrm{~m}, 3 \mathrm{H}), 7.24-7.31(\mathrm{~m}, 3 \mathrm{H}), 7.38-7.44(\mathrm{~m}, 1 \mathrm{H}), 7.55-$ $7.62(\mathrm{~m}, 1 \mathrm{H}), 7.76-7.80(\mathrm{~m}, 1 \mathrm{H}) ;{ }^{13} \mathrm{C}$ NMR $\delta 28.3,46.0,55.8,123.3,125.6,126.2,126.4$, 127.7, 128.5, 135.2, 135.7, 147.2, 162.8, 205.8; HRMS (EI) calcd for $\mathrm{C}_{16} \mathrm{H}_{14} \mathrm{O}\left(\mathrm{M}^{+}\right)$ 222.1045 , found 222.1047 .

The enantiomeric excess was determined to be $79 \%$ by chiral HPLC (Daicel CHIRALCEL ${ }^{\circledR}$ OJ-H column, hexane: $i-\mathrm{PrOH}=95: 5,0.6 \mathrm{~mL} / \mathrm{min}$; retention times: $t_{1}=14.9 \mathrm{~min}$ (major), $t_{2}$ $=30.0 \min (\operatorname{minor})$ ).<smiles>CCCC1(C)CC(=O)c2ccccc21</smiles>

3-Isopropyl-3-methylindan-1-one $(4 \mathrm{c}) .^{5}$ According to the procedure analogous to that described for $4 \mathbf{a}, 4 \mathbf{c}(30.6 \mathrm{mg}, 0.163 \mathrm{mmol}, 81 \%)$ was prepared from $3 \mathbf{c}(62.8 \mathrm{mg}, 0.20$ mmol). 4c: $[\alpha]^{27}{ }_{\mathrm{D}}+20.6\left(c 0.83, \mathrm{CHCl}_{3}\right) ;{ }^{1} \mathrm{H}$ NMR $\delta 0.60(\mathrm{~d}, J=6.9 \mathrm{~Hz}, 3 \mathrm{H}), 0.96(\mathrm{~d}, J=$ $6.6 \mathrm{~Hz}, 3 \mathrm{H}), 1.41(\mathrm{~s}, 2 \mathrm{H}), 2.03$ (sept, $J=6.8 \mathrm{~Hz}, 1 \mathrm{H}), 2.26(\mathrm{~d}, J=19.2 \mathrm{~Hz}, 1 \mathrm{H}), 2.70(\mathrm{~d}, J=$ $18.9 \mathrm{~Hz}, 1 \mathrm{H}), 7.35$ (dt, $J=0.8,7.4 \mathrm{~Hz}, 1 \mathrm{H}), 7.44(\mathrm{dd}, J=7.7,0.8 \mathrm{~Hz}, 1 \mathrm{H}), 7.55-7.63(\mathrm{~m}$, 1H), 7.66-7.71 (m, 1H); ${ }^{13} \mathrm{C}$ NMR $\delta 18.1,18.4,27.3,36.7,45.6,46.4,123.2,124.0,127.3$, 134.7, 136.2, 162.7, 206.4; HRMS (EI) calcd for $\left(\mathrm{M}^{+}\right)$188.12001, found 188.1200.

(4) [26466-19-3] (racemate)

(5) [2977-29-9] (racemate) 
The enantiomeric excess was determined to be $94 \%$ by chiral GC (Chiraldex G-BP column, $100{ }^{\circ} \mathrm{C}(5 \mathrm{~min})$ then $5{ }^{\circ} \mathrm{C} / \mathrm{min}$ to $120^{\circ} \mathrm{C}(22 \mathrm{~min})$; retention times: $t_{1}=20.3 \mathrm{~min}$ (major), $t_{2}$ $=21.7 \mathrm{~min}$ (minor)).<smiles>Cc1ccc2c(c1)C(=O)CC2(C)CCCO</smiles>

(S)-3-(3-Benzyloxypropyl)-3,5-dimethylindan-1-one (9). According to the procedure analogous to that described for $\mathbf{4 a}, \mathbf{9}(155 \mathrm{mg}, 0.503 \mathrm{mmol}, 84 \%)$ was prepared from 3c (261 mg, $0.60 \mathrm{mmol}) .9$ : $[\alpha]^{27}{ }_{\mathrm{D}}-4.2\left(c 1.0, \mathrm{CHCl}_{3}\right) ;{ }^{1} \mathrm{H}$ NMR $\delta 1.18-1.36(\mathrm{~m}, 1 \mathrm{H}), 1.40(\mathrm{~s}$, $3 \mathrm{H}), 1.48-1.64(\mathrm{~m}, 1 \mathrm{H}), 1.66-1.88(\mathrm{~m}, 2 \mathrm{H}), 2.44(\mathrm{~s}, 3 \mathrm{H}), 2.44(\mathrm{~d}, J=18.9 \mathrm{~Hz}, 1 \mathrm{H}), 2.66(\mathrm{~d}$, $J=18.9 \mathrm{~Hz}, 1 \mathrm{H}), 3.40(\mathrm{t}, J=6.3 \mathrm{~Hz}, 2 \mathrm{H}), 4.44(\mathrm{~s}, 2 \mathrm{H}), 7.14-7.19(\mathrm{~m}, 1 \mathrm{H}), 7.22-7.37$ (m, $6 \mathrm{H}), 7.59(\mathrm{~d}, J=7.8 \mathrm{~Hz}, 1 \mathrm{H}) ;{ }^{13} \mathrm{C}$ NMR $\delta 22.3,25.5,28.4,38.6,41.6,50.3,70.4,72.9$, $123.2,124.1,127.51,127.54,128.3,128.7,133.8,138.3,146.0,163.0,205.3$; HRMS (FAB) calcd for $\mathrm{C}_{21} \mathrm{H}_{25} \mathrm{O}_{2}\left(\mathrm{M}^{+}+\mathrm{H}\right) 309.1855$, found 309.1857 .

The enantiomeric excess was determined to be $94 \%$ by chiral HPLC (Daicel CHIRALCEL ${ }^{\circledR}$ OJ-H, hexane: $i-\mathrm{PrOH}=97: 3,0.6 \mathrm{~mL} / \mathrm{min})$; retention times: $t_{1}=42.9 \mathrm{~min}(S), t_{2}=48.0 \mathrm{~min}$ $(R))$.

\section{Synthesis of (-)- $\alpha$-Herbertenol (13)}
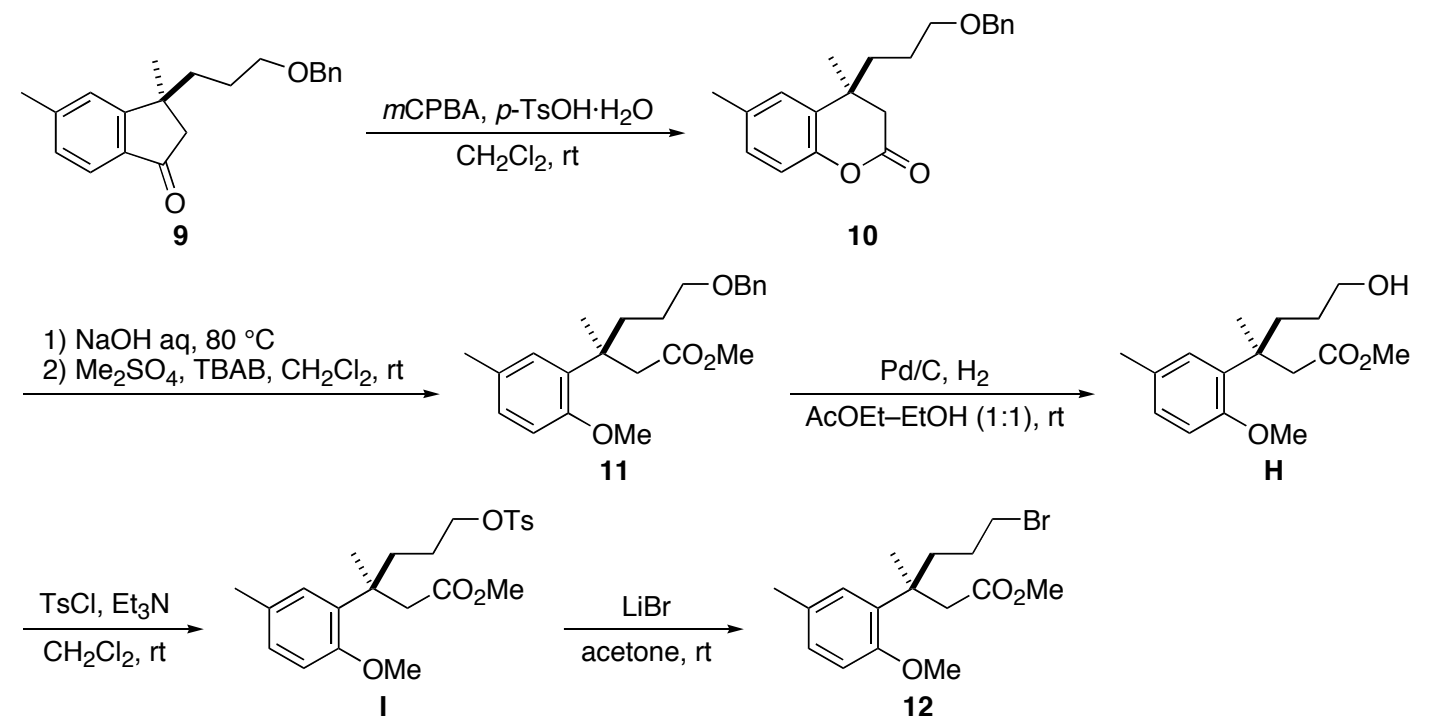
(S)-4-(3-Benzyloxypropyl)-4,6-dimethylchroman-2-one (10). A mixture of 9 (155 mg, $0.503 \mathrm{mmol}), m \mathrm{CPBA}(77 \%, 1.14 \mathrm{~g}, 5.09 \mathrm{mmol})$, and $p-\mathrm{TsOH} \cdot \mathrm{H}_{2} \mathrm{O}(174 \mathrm{mg}, 0.916 \mathrm{mmol})$ in $\mathrm{CH}_{2} \mathrm{Cl}_{2}(4.0 \mathrm{~mL})$ was stirred at room temperature for $40 \mathrm{~h}$. The mixture was diluted with $\mathrm{CH}_{2} \mathrm{Cl}_{2}$ and washed with saturated $\mathrm{Na}_{2} \mathrm{SO}_{3}$ aqueous solution, saturated $\mathrm{NaHCO}_{3}$ aqueous solution, and brine. The organic phase was dried over $\mathrm{MgSO}_{4}$ and concentrated. The residue was purified by preparative thin-layer chromatography (hexane:AcOEt $=6: 1$ ) to afford 10 (107 mg, $0.330 \mathrm{mmol}, 66 \%)$ : ${ }^{1} \mathrm{H}$ NMR $\delta 1.35$ (s, 3H), 1.40-1.54 (m, 1H), 1.55$1.74(\mathrm{~m}, 3 \mathrm{H}), 2.33(\mathrm{~s}, 3 \mathrm{H}), 2.56(\mathrm{~d}, J=15.3 \mathrm{~Hz}, 1 \mathrm{H}), 2.69$ (d, $J=15.9 \mathrm{~Hz}, 1 \mathrm{H}), 3.37-3.43$ $(\mathrm{m}, 2 \mathrm{H}), 4.45(\mathrm{~s}, 2 \mathrm{H}), 6.91-6.98(\mathrm{~m}, 1 \mathrm{H}), 7.00-7.09(\mathrm{~m}, 2 \mathrm{H}), 7.22-7.38(\mathrm{~m}, 5 \mathrm{H}) ;{ }^{13} \mathrm{C} \mathrm{NMR} \delta$ 21.0, 24.6, 25.2, 36.1, 36.6, 41.9, 70.2, 73.0, 116.9, 125.8, 127.55, 127.60, 128.3, 128.7, 129.7, 134.0, 138.3, 148.8, 168.4; HRMS (FAB) calcd for $\mathrm{C}_{21} \mathrm{H}_{25} \mathrm{O}_{3}\left(\mathrm{M}^{+}+\mathrm{H}\right)$ 325.1804, found 325.1806 .

(S)-Methyl 6-benzyloxy-3-(2-methoxy-5-methylphenyl)-3-methylhexanoate (11). A mixture of $10(107 \mathrm{mg}, 0.330 \mathrm{mmol})$ and $\mathrm{NaOH}$ aq $(4.2 \mathrm{M}, 1.0 \mathrm{~mL})$ was stirred at $80{ }^{\circ} \mathrm{C}$ for $3 \mathrm{~h}$. To the reaction mixture was added $\mathrm{CH}_{2} \mathrm{Cl}_{2}(1.0 \mathrm{~mL}), \mathrm{Bu}_{4} \mathrm{NBr}(63.8 \mathrm{mg}, 0.198 \mathrm{mmol})$, $\mathrm{Me}_{2} \mathrm{SO}_{4}(916 \mathrm{mg}, 7.26 \mathrm{mmol})$ at $0{ }^{\circ} \mathrm{C}$, then the mixture was stirred at room temperature for 2 days. The organic layer was separated and the aqueous layer was extracted with $\mathrm{CH}_{2} \mathrm{Cl}_{2}$. The combined organic layer was dried over $\mathrm{MgSO}_{4}$ and concentrated. The residue was purified by preparative thin-layer chromatography (hexane:AcOEt $=6: 1)$ to afford $11(101$ $\mathrm{mg}, 0.273 \mathrm{mmol}, 83 \%):{ }^{1} \mathrm{H}$ NMR $\delta 1.10-1.28$ (m, 1H), 1.38-1.67 (m, 2H), 1.47 (s, 3H), 2.20-2.33 (m, 1H), $2.26(\mathrm{~s}, 3 \mathrm{H}), 2.66(\mathrm{~d}, J=14.1 \mathrm{~Hz}, 1 \mathrm{H}), 3.13(\mathrm{~d}, J=14.1 \mathrm{~Hz}, 1 \mathrm{H}), 3.37(\mathrm{t}$, $J=6.9 \mathrm{~Hz}, 2 \mathrm{H}), 3.47(\mathrm{~s}, 3 \mathrm{H}), 3.77(\mathrm{~s}, 3 \mathrm{H}), 4.42(\mathrm{~s}, 2 \mathrm{H}), 6.73(\mathrm{~d}, J=8.7 \mathrm{~Hz}, 1 \mathrm{H}), 6.95-7.01$ $(\mathrm{m}, 2 \mathrm{H}), 7.22-7.36(\mathrm{~m}, 5 \mathrm{H}) ;{ }^{13} \mathrm{C} \mathrm{NMR} \delta 20.8,25.09,25.12,36.0,40.2,44.7,50.9,55.2$, $71.0,72.7,111.2,127.4,127.5,127.7,128.2,129.1,132.7,138.6,156.0,172.7$ [one $\mathrm{sp}^{2}$ carbon signal is missing due to overlapping]; HRMS (FAB) calcd for $\mathrm{C}_{23} \mathrm{H}_{30} \mathrm{O}_{4}\left(\mathrm{M}^{+}\right)$ 370.2144 , found 370.2148 .

(S)-Methyl 6-hydroxy-3-(2-methoxy-5-methylphenyl)-3-methylhexanoate $\quad(\mathrm{H}) . \quad \mathrm{A}$ mixture of $11(101 \mathrm{mg}, 0.273 \mathrm{mmol})$ and $\mathrm{Pd} / \mathrm{C}(5 \%, 51 \mathrm{mg})$ in $\mathrm{MeOH}-\operatorname{AcOEt}(1: 1,6.0$ $\mathrm{mL}$ ) was stirred under hydrogen atmosphere at room temperature for $12 \mathrm{~h}$. The reaction mixture was filtered (Florisil ${ }^{\circledR}$, AcOEt) and concentrated. The residue was purified by 
preparative thin-layer chromatography (hexane:AcOEt $=2: 1)$ to afford $\mathbf{H}(56.9 \mathrm{mg}, 0.203$ mmol, 74\%): ${ }^{1} \mathrm{H}$ NMR $\delta 1.03-1.18(\mathrm{~m}, 1 \mathrm{H}), 1.33-1.46(\mathrm{~m}, 1 \mathrm{H}), 1.40(\mathrm{~s}, 1 \mathrm{H}), 1.47$ (s, 3H), $1.60(\mathrm{dt}, J=4.8,12.6 \mathrm{~Hz}, 1 \mathrm{H}), 2.26(\mathrm{~s}, 3 \mathrm{H}), 2.27(\mathrm{dt}, J=4.4,12.7 \mathrm{~Hz}, 1 \mathrm{H}), 2.68(\mathrm{~d}, J=$ $13.8 \mathrm{~Hz}, 1 \mathrm{H}$ ), 3.09 (d, $J=13.8 \mathrm{~Hz}, 1 \mathrm{H}), 3.47$ (s, 3H), 3.51 (t, $J=6.5 \mathrm{~Hz}, 2 \mathrm{H}), 3.80$ (s, 3H), $6.75(\mathrm{t}, J=8.1 \mathrm{~Hz}, 1 \mathrm{H}), 6.95-7.01(\mathrm{~m}, 2 \mathrm{H}) ;{ }^{13} \mathrm{C} \mathrm{NMR} \delta 20.8,25.1,28.2,35.5,40.2,44.6$, 50.9, 55.2, 63.4, 111.2, 127.7, 129.0, 129.2, 132.6, 155.9, 172.6; HRMS (EI) calcd for $\mathrm{C}_{16} \mathrm{H}_{24} \mathrm{O}_{4}\left(\mathrm{M}^{+}\right)$280.1675, found 280.1674 .

\section{(S)-Methyl}

3-(2-methoxy-5-methylphenyl)-3-methyl-6-(toluene-4-sulfonyloxy)-

hexanoate (I). To a solution of $\mathbf{H}(56.9 \mathrm{mg}, 0.203 \mathrm{mmol})$ in $\mathrm{CH}_{2} \mathrm{Cl}_{2}(2.0 \mathrm{~mL})$ was added $\mathrm{Et}_{3} \mathrm{~N}(57.9 \mu \mathrm{L}), \mathrm{TsCl}(77.4 \mathrm{mg}, 0.406 \mathrm{mmol})$, and DMAP $(7.4 \mathrm{mg}, 61 \mu \mathrm{mol})$, and the mixture was stirred at room temperature for $3 \mathrm{~h}$. The reaction mixture was diluted with $\mathrm{CH}_{2} \mathrm{Cl}_{2}$ and washed with $\mathrm{H}_{2} \mathrm{O}$, saturated $\mathrm{NH}_{4} \mathrm{Cl}$ aqueous solution, and brine. The organic layer was dried over $\mathrm{MgSO}_{4}$ and concentrated to give crude $\mathbf{I}$.

(S)-Methyl 6-bromo-3-(2-methoxy-5-methylphenyl)-3-methylhexanoate (12). To a solution of crude $\mathbf{I}$ in acetone $(3.0 \mathrm{~mL})$ was added $\mathrm{LiBr}(176 \mathrm{mg}, 2.03 \mathrm{mmol})$, and the mixture was stirred at room temperature for $12 \mathrm{~h}$. The reaction mixture was concentrated, and the residue was diluted with $\mathrm{Et}_{2} \mathrm{O}$, washed with $\mathrm{H}_{2} \mathrm{O}$ and brine, dried over $\mathrm{MgSO}_{4}$, and concentrated. The residue was purified by preparative thin-layer chromatography (hexane:AcOEt = 8:1) to afford $12(67.0 \mathrm{mg}, 0.195 \mathrm{mmol}, 96 \%$ from $\mathbf{H}):[\alpha]^{25}{ }_{\mathrm{D}}+16.2(c$ 1.8, $\left.\mathrm{CHCl}_{3}\right) ;{ }^{1} \mathrm{H}$ NMR $\delta 1.31-1.50(\mathrm{~m}, 1 \mathrm{H}), 1.47(\mathrm{~s}, 3 \mathrm{H}), 1.59-1.75(\mathrm{~m}, 2 \mathrm{H}), 2.27(\mathrm{~s}, 3 \mathrm{H})$, 2.33-2.48 (m, 1H), $2.67(\mathrm{~d}, J=13.8 \mathrm{~Hz}, 1 \mathrm{H}), 3.09(\mathrm{~d}, J=13.8 \mathrm{~Hz}, 1 \mathrm{H}), 3.29(\mathrm{t}, J=6.6 \mathrm{~Hz}$, 2H), $3.48(\mathrm{~s}, 3 \mathrm{H}), 3.80(\mathrm{~s}, 3 \mathrm{H}), 6.75(\mathrm{~d}, J=8.4 \mathrm{~Hz}, 1 \mathrm{H}), 6.92-7.03(\mathrm{~m}, 2 \mathrm{H}) ;{ }^{13} \mathrm{C}$ NMR $\delta$ 20.9, 25.1, 28.5, 34.6, 38.2, 40.1, 44.6, 51.0, 55.2, 111.2, 127.9, 129.0, 129.2, 132.2, 155.9, 172.5; HRMS (EI) calcd for $\mathrm{C}_{16} \mathrm{H}_{23} \mathrm{BrO}_{3}\left(\mathrm{M}^{+}\right) 342.0831$, found 342.0830 .

The enantiomeric excess was determined to be $94 \%$ by chiral HPLC (Daicel CHIRALCEL ${ }^{\circledR}$ OD-H, hexane: $i-\mathrm{PrOH}=99.5: 0.5,0.6 \mathrm{~mL} / \mathrm{min})$; retention times: $t_{1}=16.3 \mathrm{~min}(S), t_{2}=20.5$ $\min (R))$. 
(-)- $\alpha$-Herbertenol (13). ${ }^{6}$ The bromoester 12, which was prepared from 9 of $97 \%$ ee, was transformed to $\mathbf{1 3}$ according to the procedure reported by Mukherjee. ${ }^{7}$ Measurement of the optical rotation of $13\left([\alpha]^{28}-43.3\left(c 0.15, \mathrm{CHCl}_{3}\right) ;\right.$ lit. $^{8}[\alpha]_{\mathrm{D}}^{25}-55.0$ (c 1.8, $\left.\left.\mathrm{CHCl}_{3}\right)\right)$ established that the stereochemistry was that of the natural enantiomer. The absolute stereochemistry was also confirmed by chiral HPLC analysis of methyl ether of $\mathbf{1 3}$ by comparison with the HPLC data obtained by Profs. Kita and Fujioka. ${ }^{9}$ The enantiomeric excess was determined to be $92 \%$ by chiral HPLC (Daicel CHIRALCEL ${ }^{\circledR}$ OD-H, hexane:i$\operatorname{PrOH}=99.5: 0.5,0.41 \mathrm{~mL} / \mathrm{min})$; retention times: $\left.t_{1}=13.4 \mathrm{~min}(R), t_{2}=15.5 \mathrm{~min}(S)\right)$.

\section{Deuterium-Labeling Experiments}

When the reaction of 3a was carried out in 1,4-dioxane- $\mathrm{D}_{2} \mathrm{O}$, the produced indanone incorporated a deuterium not at the methyl group but at the 4-position (17\% D) (eq S1). The enolizable methylene group $\alpha$ to the carbonyl group was also deuterated under the basic reaction conditions. This result indicated that a 1,4-Rh shift process occurred with alkylrhodium species $\mathbf{5}$ to generate arylrhodium prior to the protonolysis.

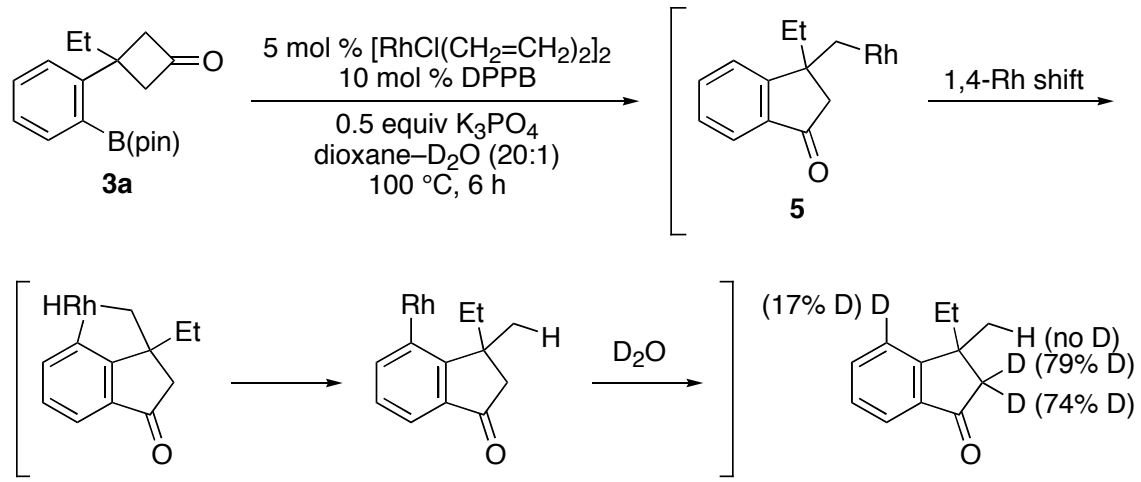

The reaction with 3a- $d_{4}\left(97 \%\right.$ D) in 1,4-dioxane- $\mathrm{D}_{2} \mathrm{O}$ resulted in deuterium enrichment both at the methyl group and at the 4-position (eq S2). The former enrichment could be

(6) [81784-10-3]

(7) Paul, T.; Pal, A.; Gupta, P. D.; Mukherjee, D. Tetrahedron Lett. 2003, 44, 737.

(8) Matsuo, A.; Yuki, S.; Nakayama, M. J. Chem. Soc., Perkin Trans. 1 1986, 701.

(9) Kita, Y.; Futamura, J.; Ohba, Y.; Sawama, Y.; Ganesh, J. K.; Fujioka, H. J. Org. Chem. 2003, 68, 5917. We thank Profs. Kita and Fujioka (Osaka University) for providing the HPLC data of methyl ether of $\mathbf{1 3 .}$ 
accounted for by rather unusual $1,3-\mathrm{Rh}$ shift. The ratio of the $1,4-/ 1,3-\mathrm{Rh}$ shift for $3 \mathbf{a}-d_{4}$ was roughly estimated to be $2 / 3$.
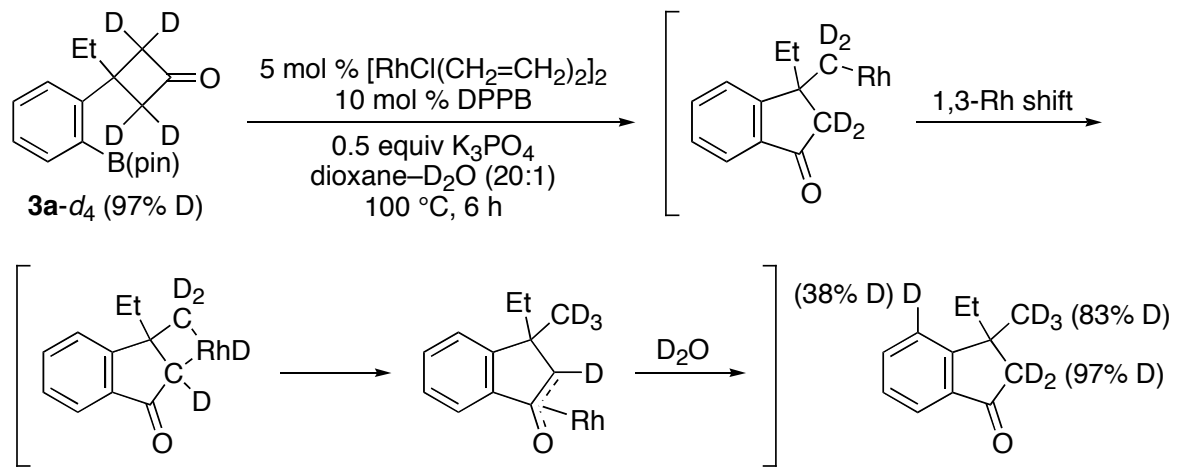

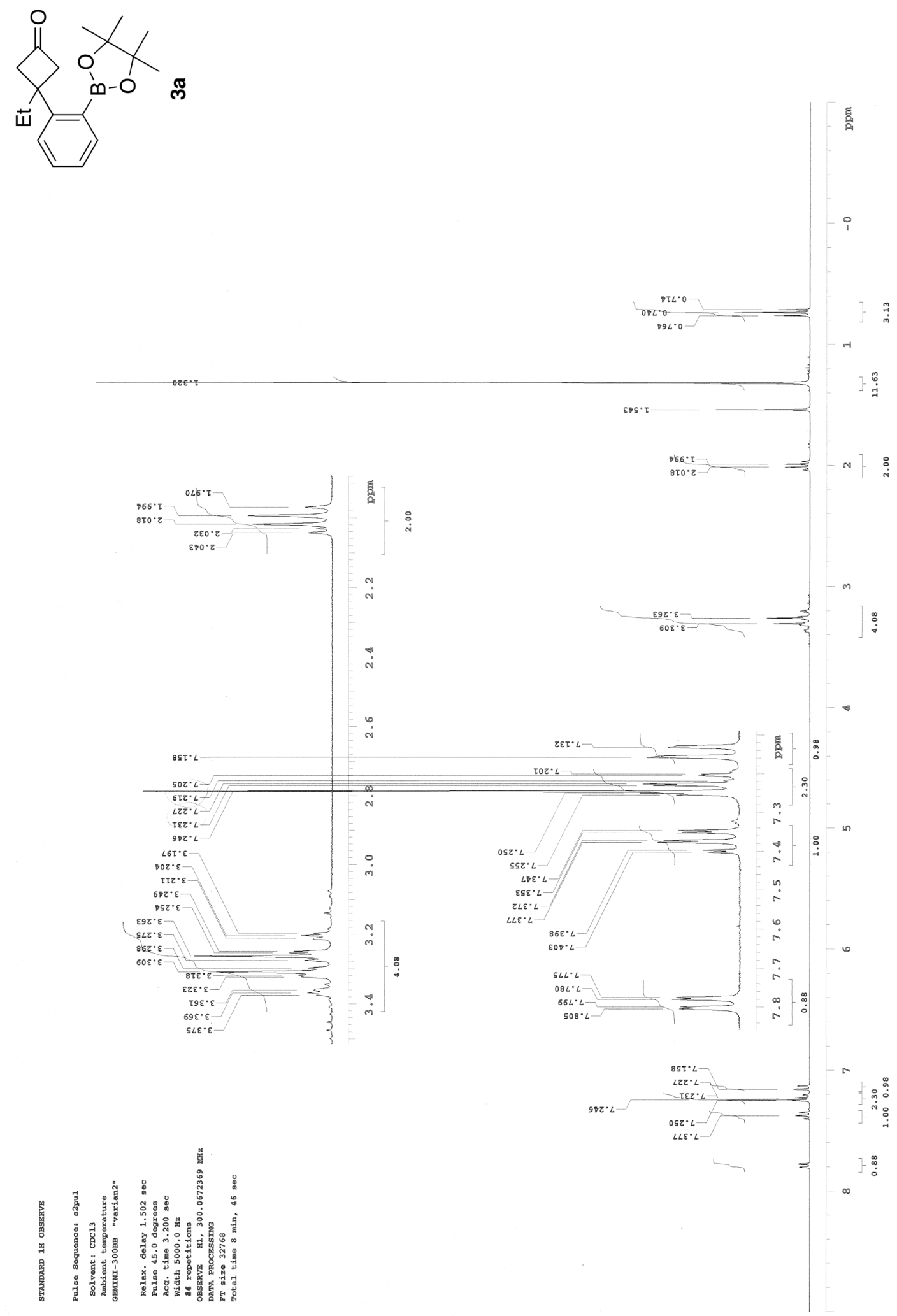

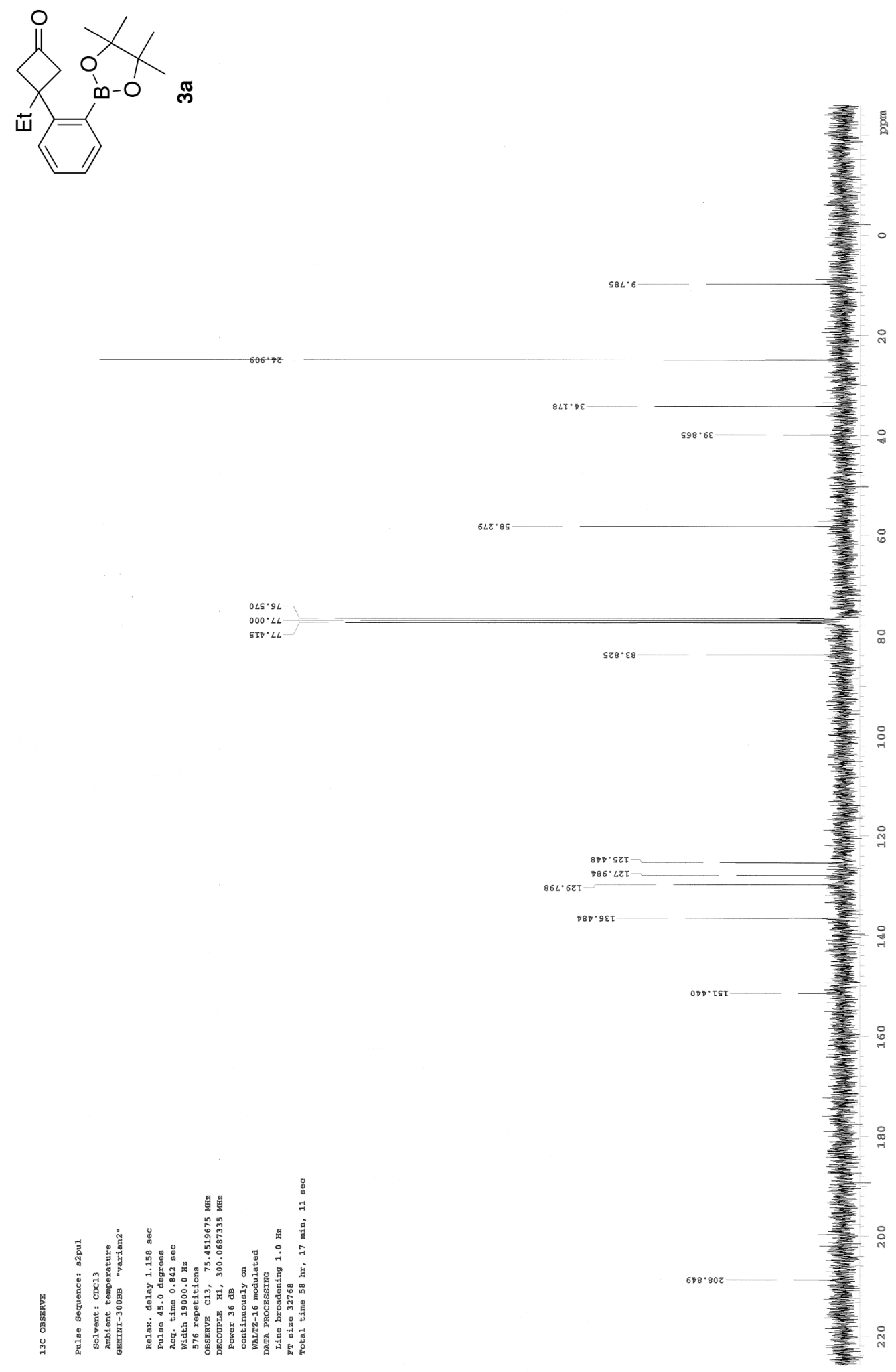

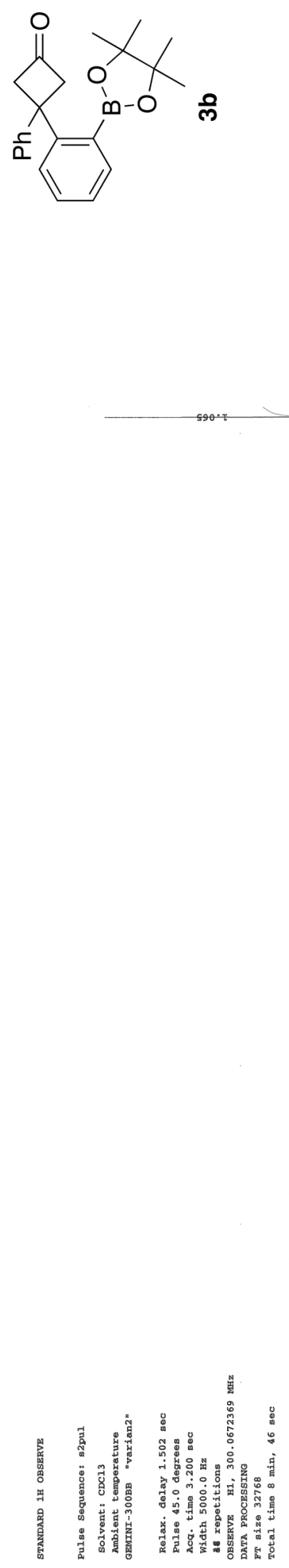

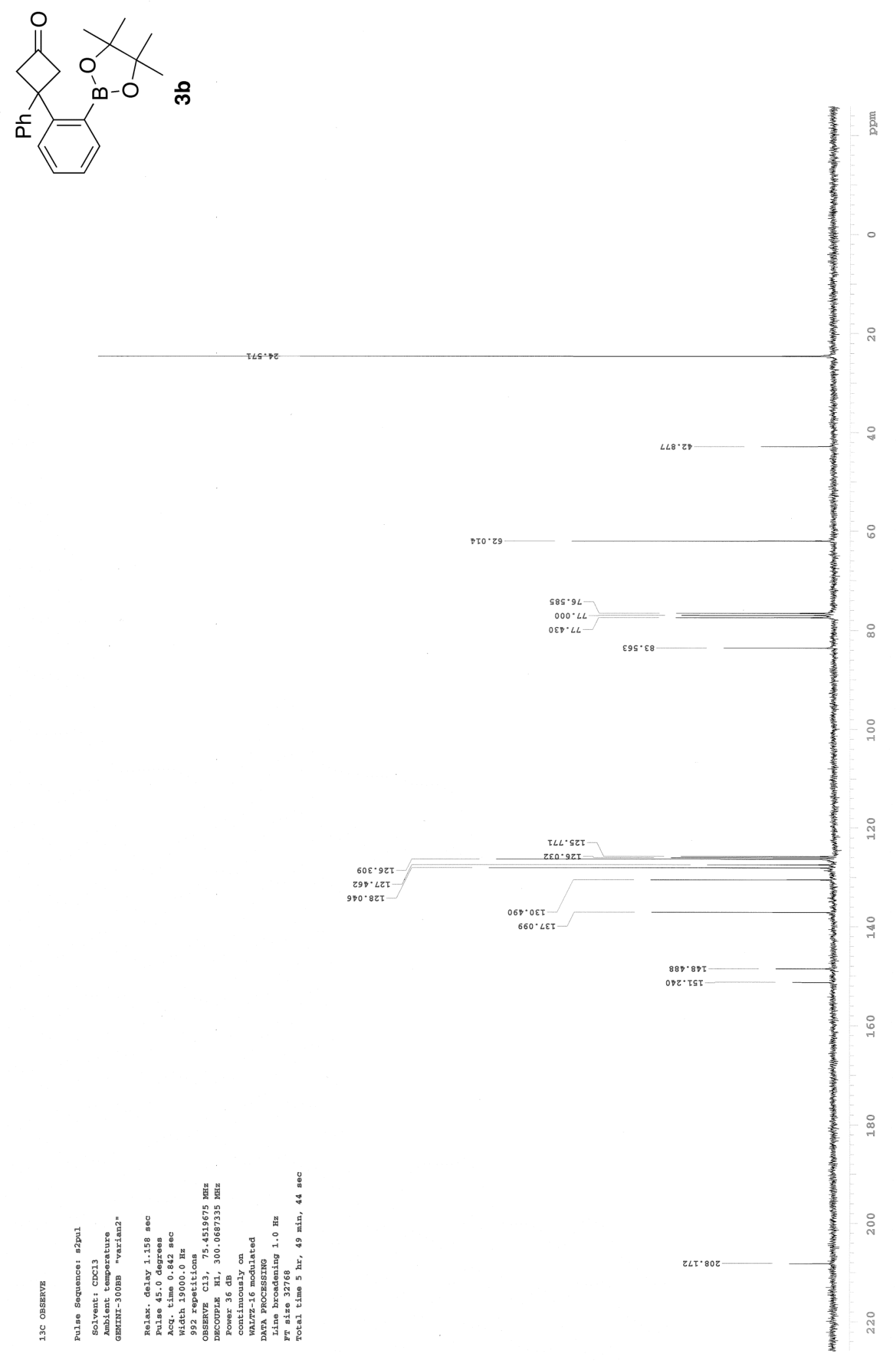

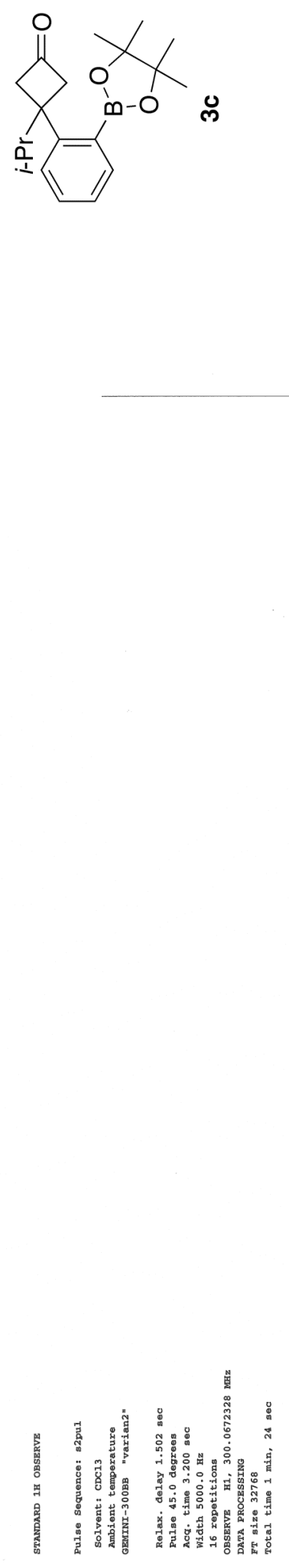

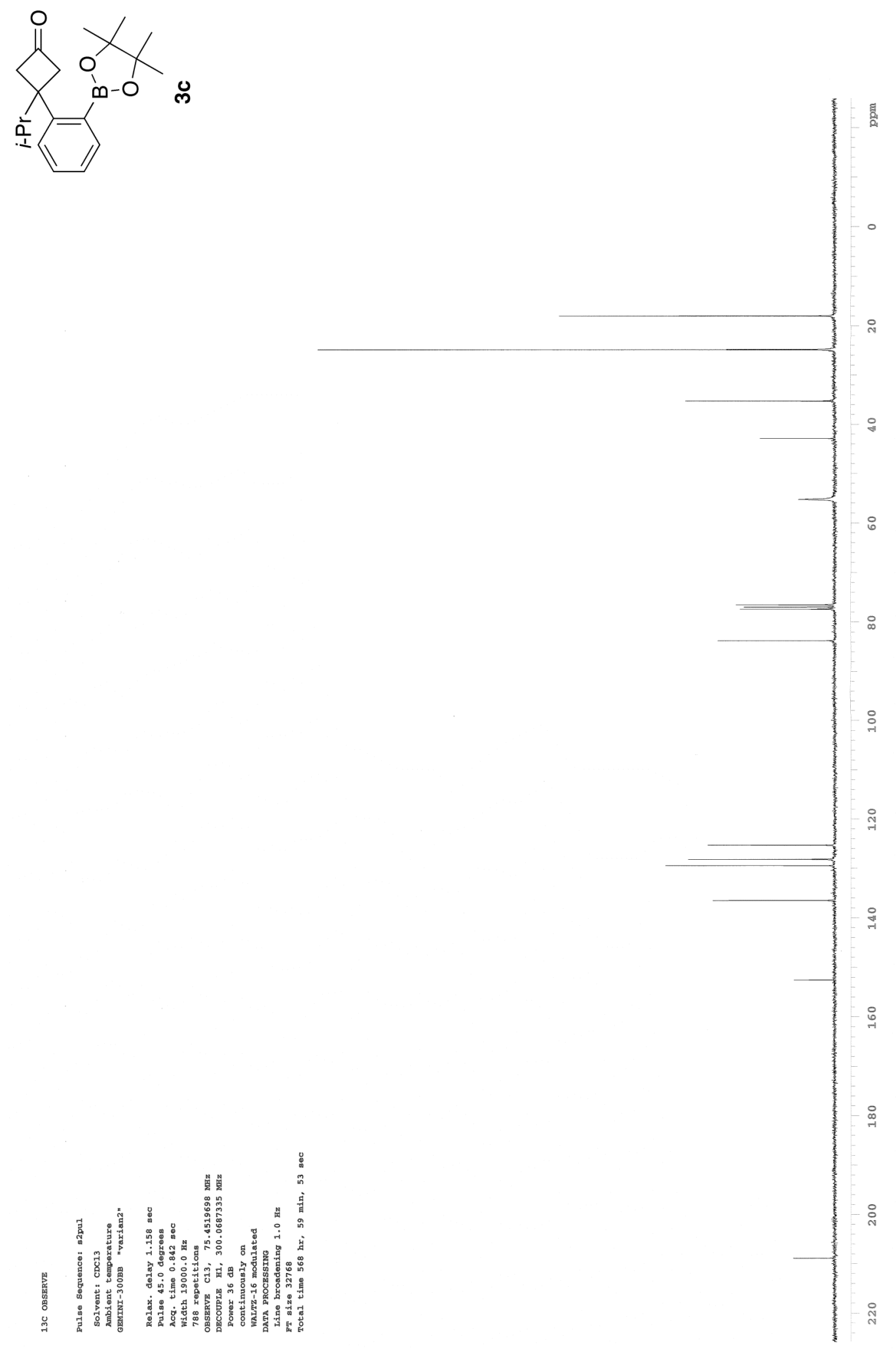

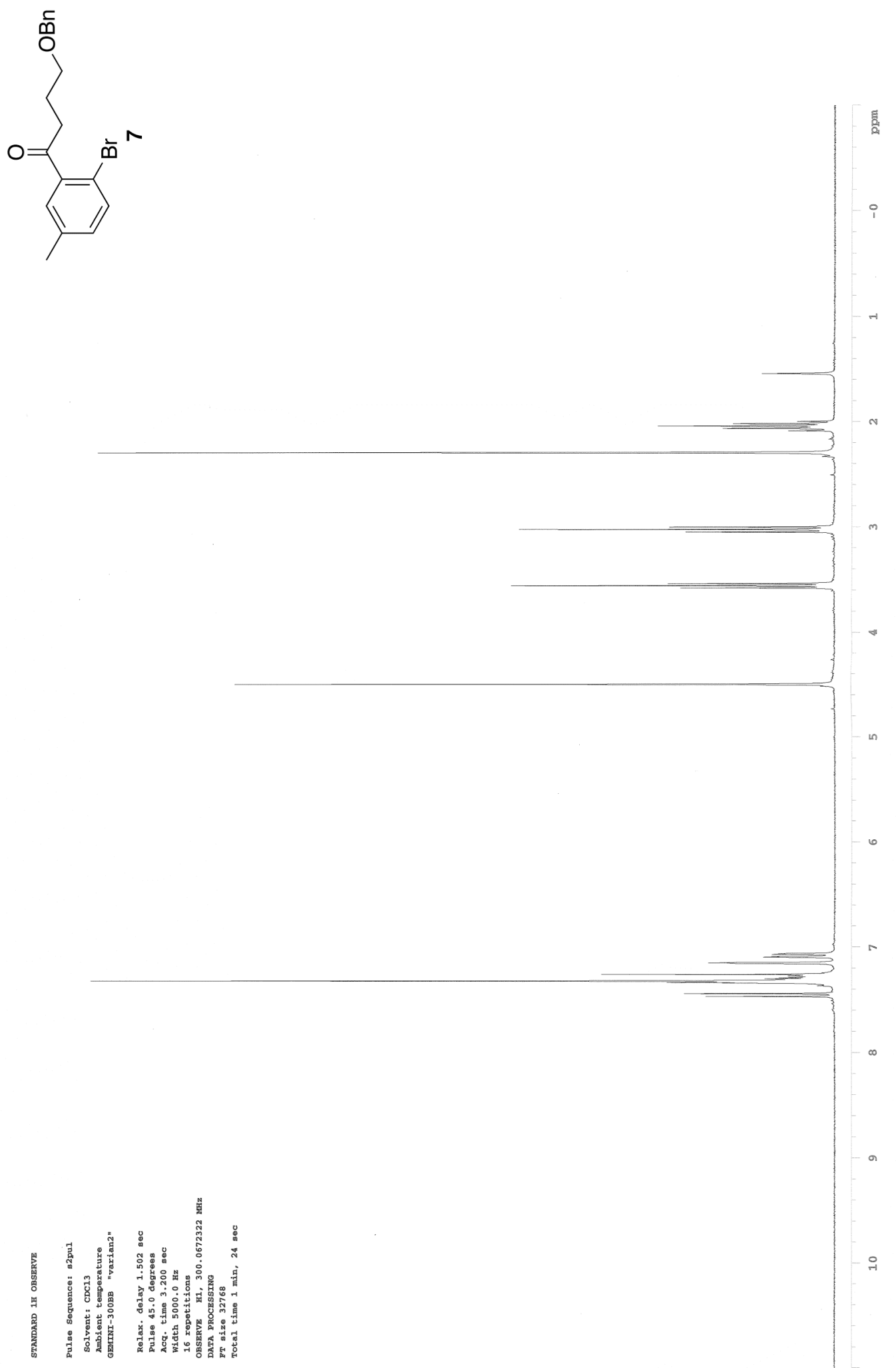

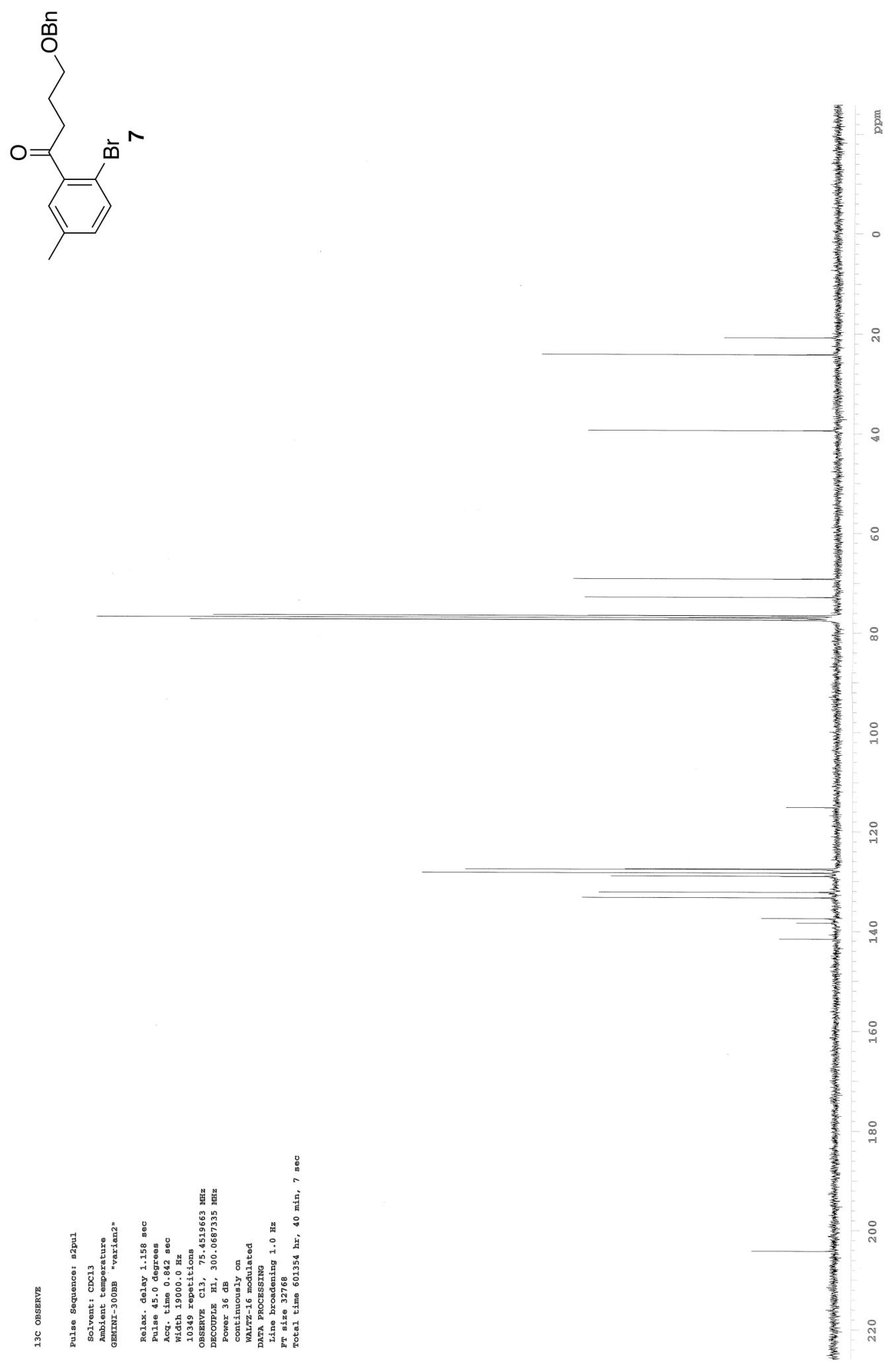

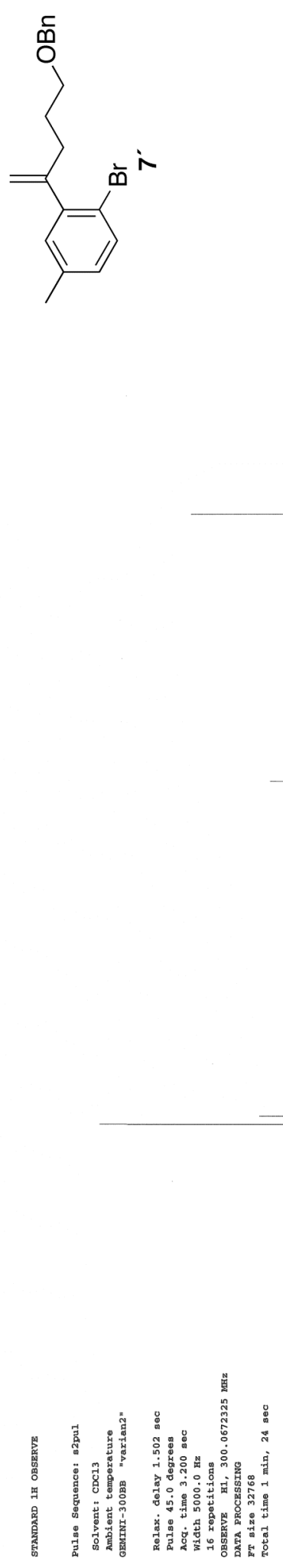

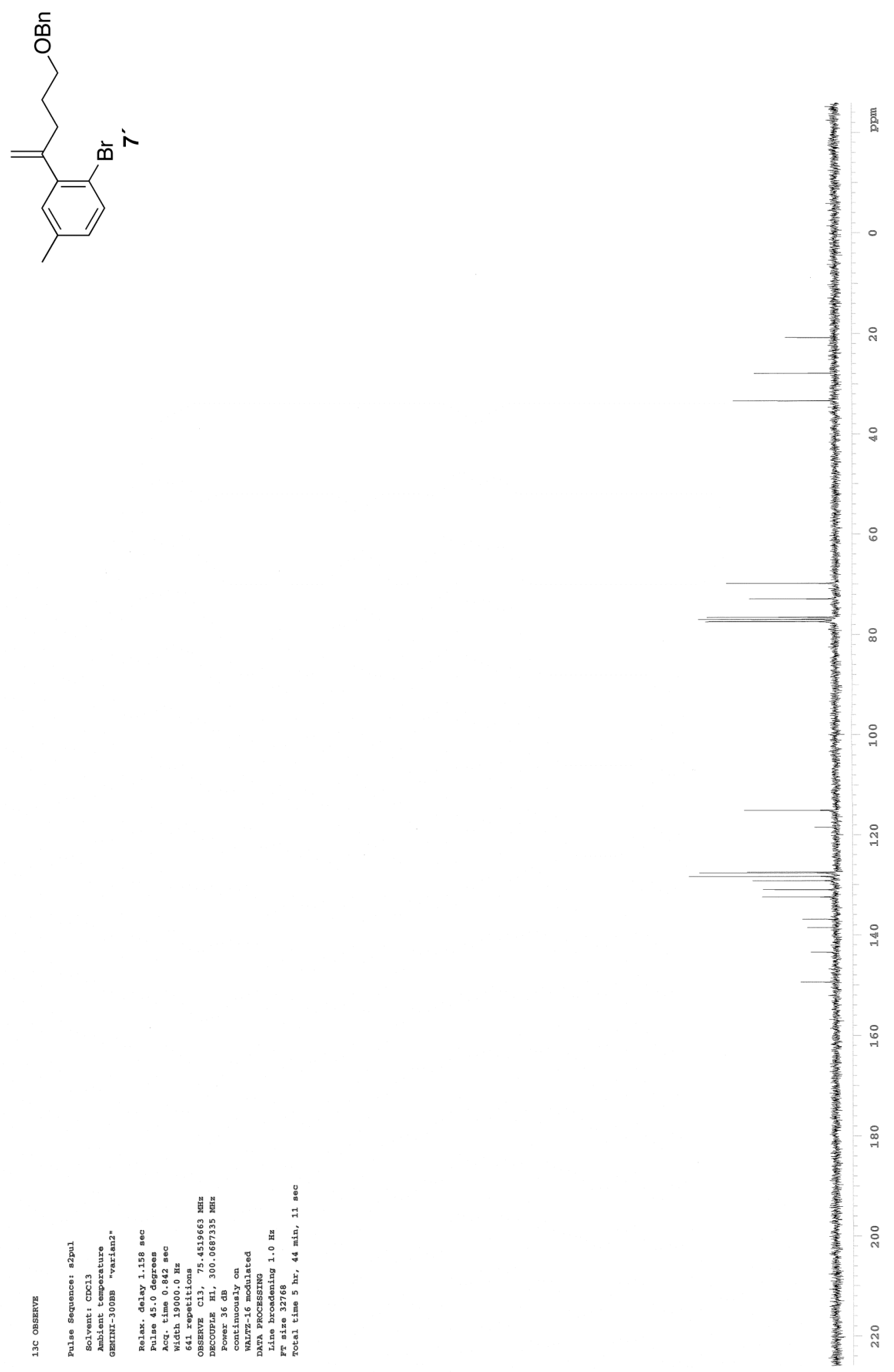

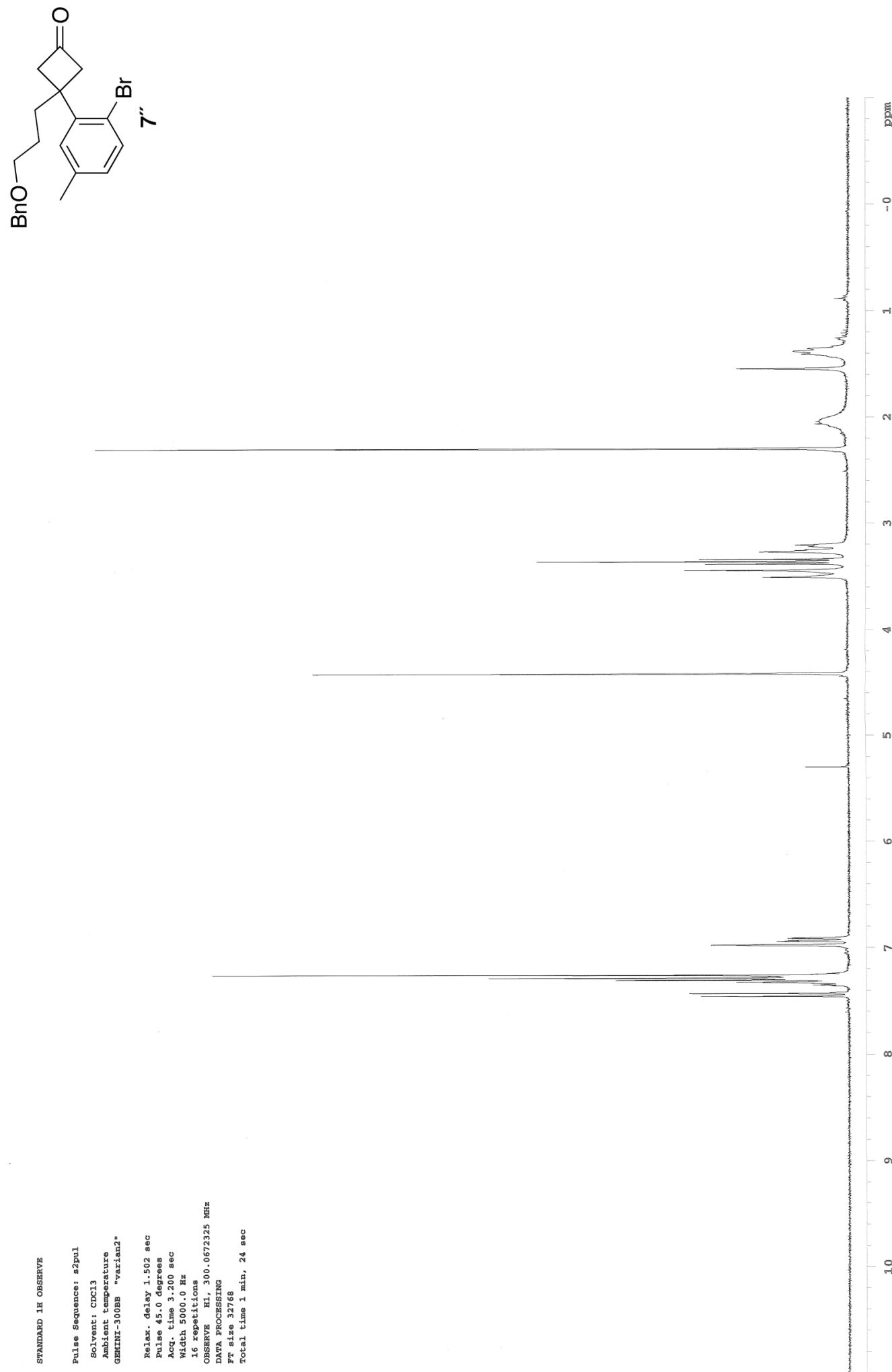

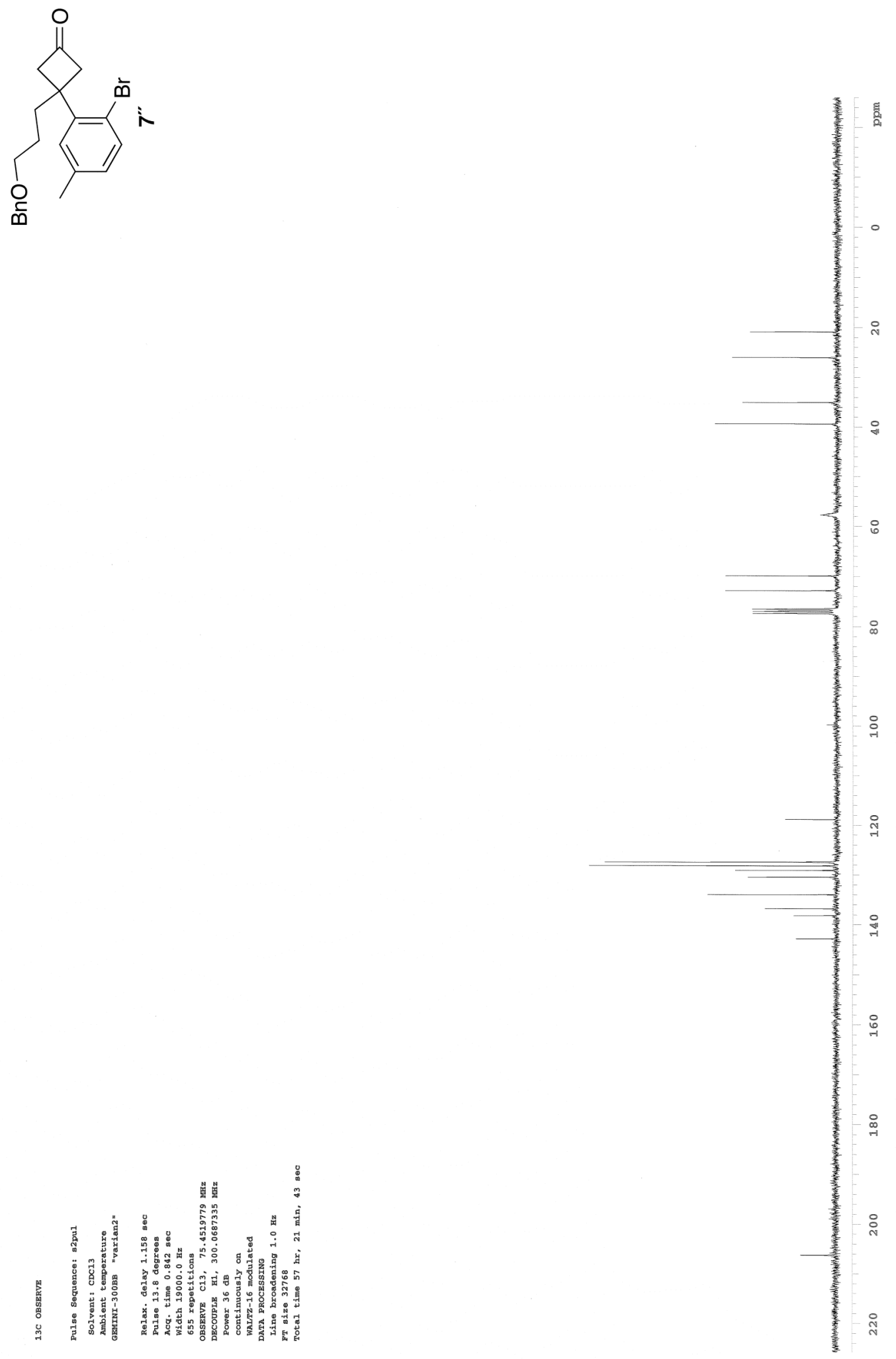

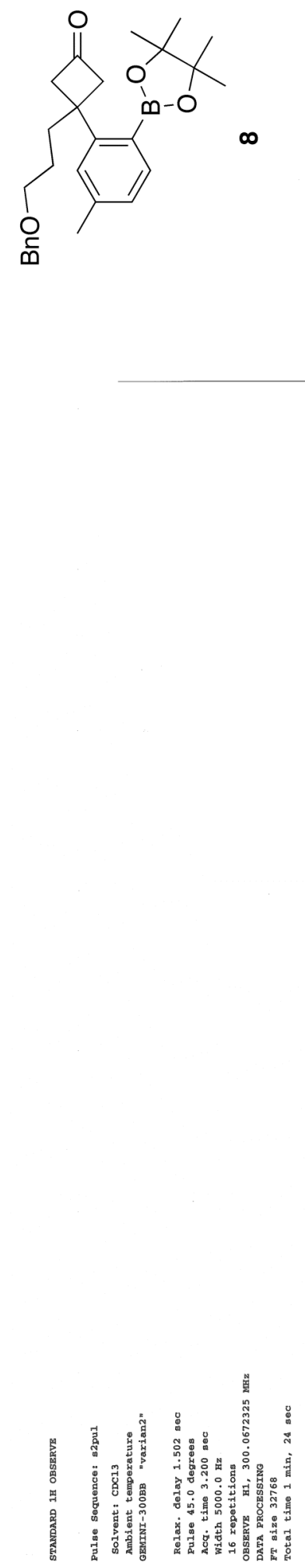

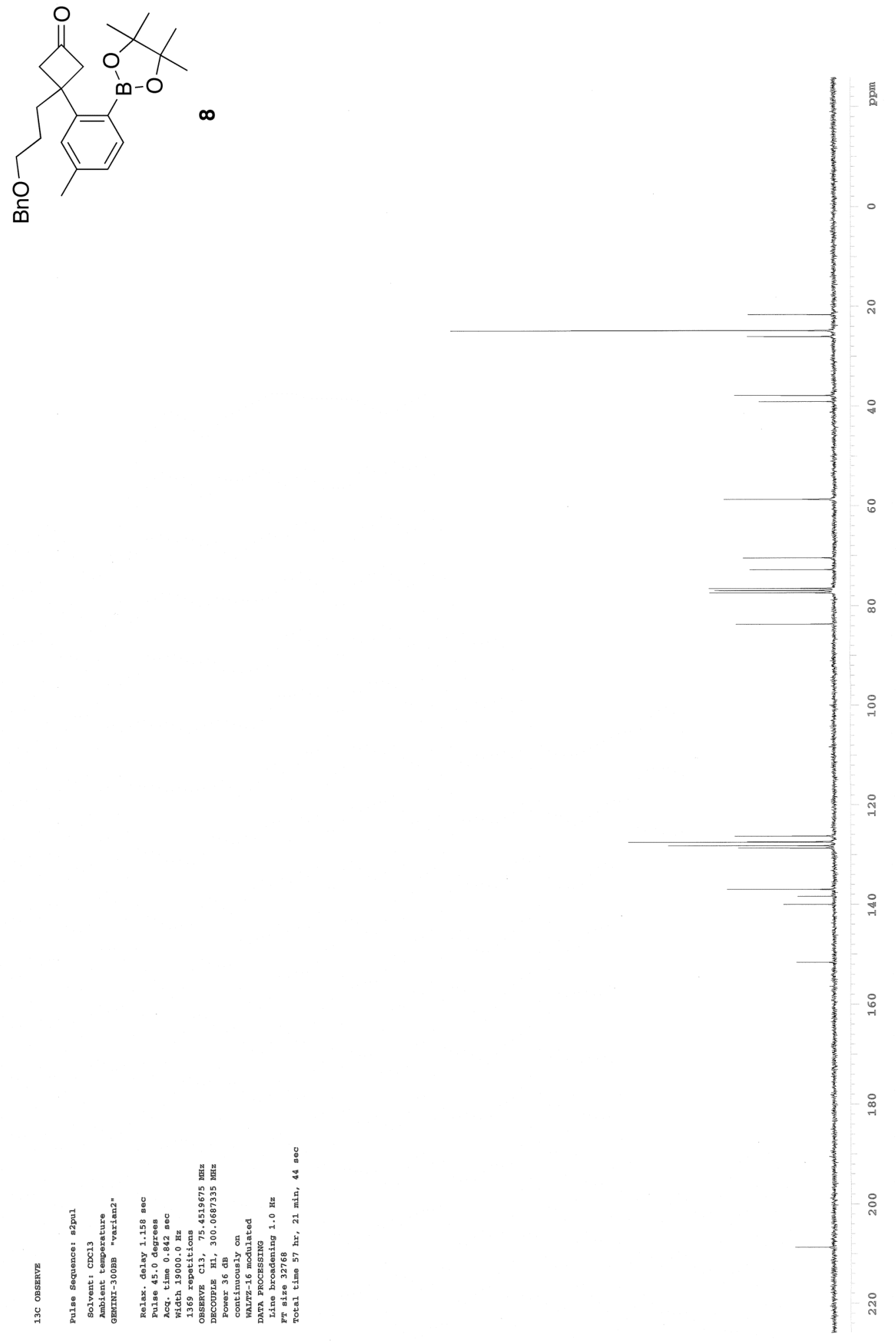

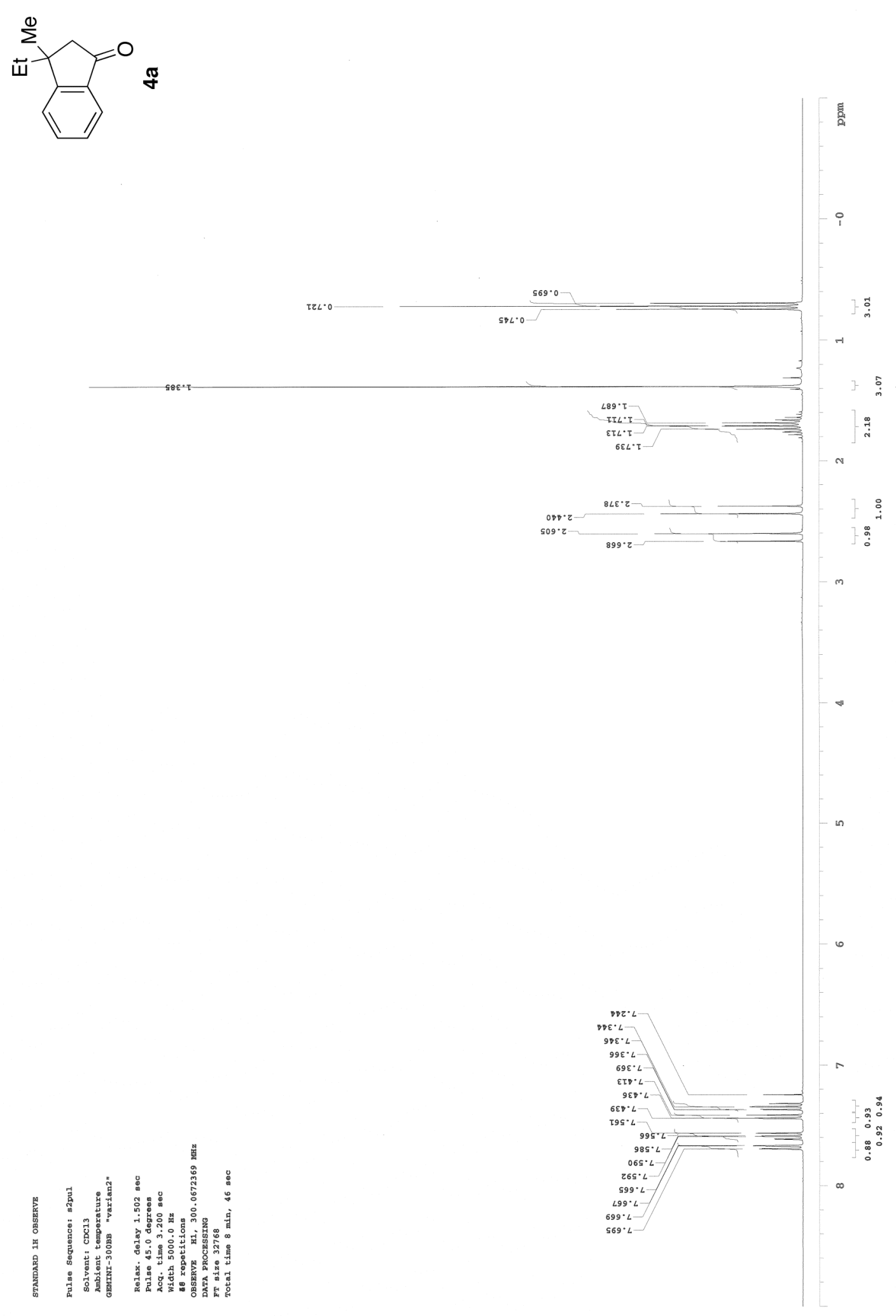

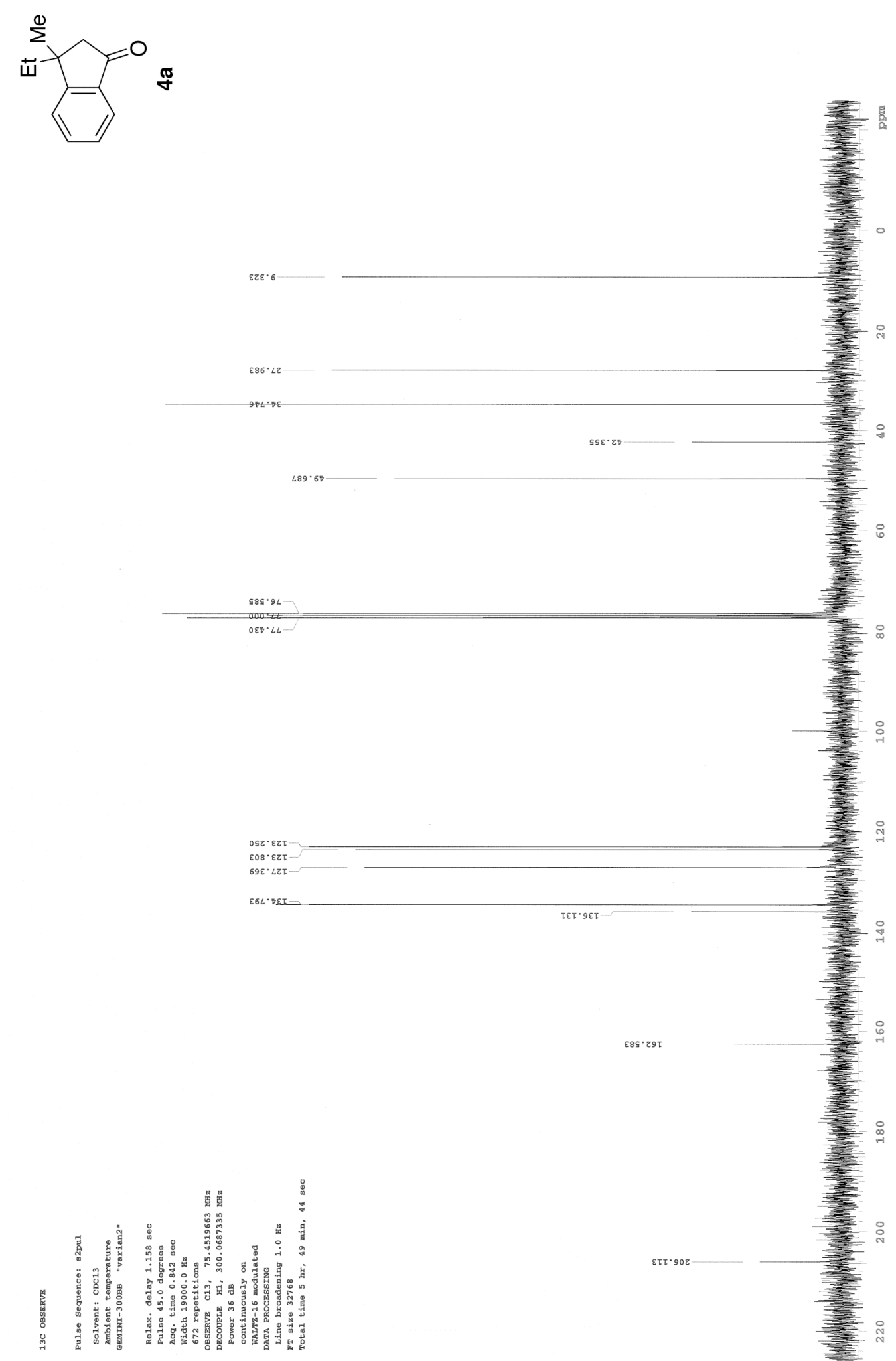


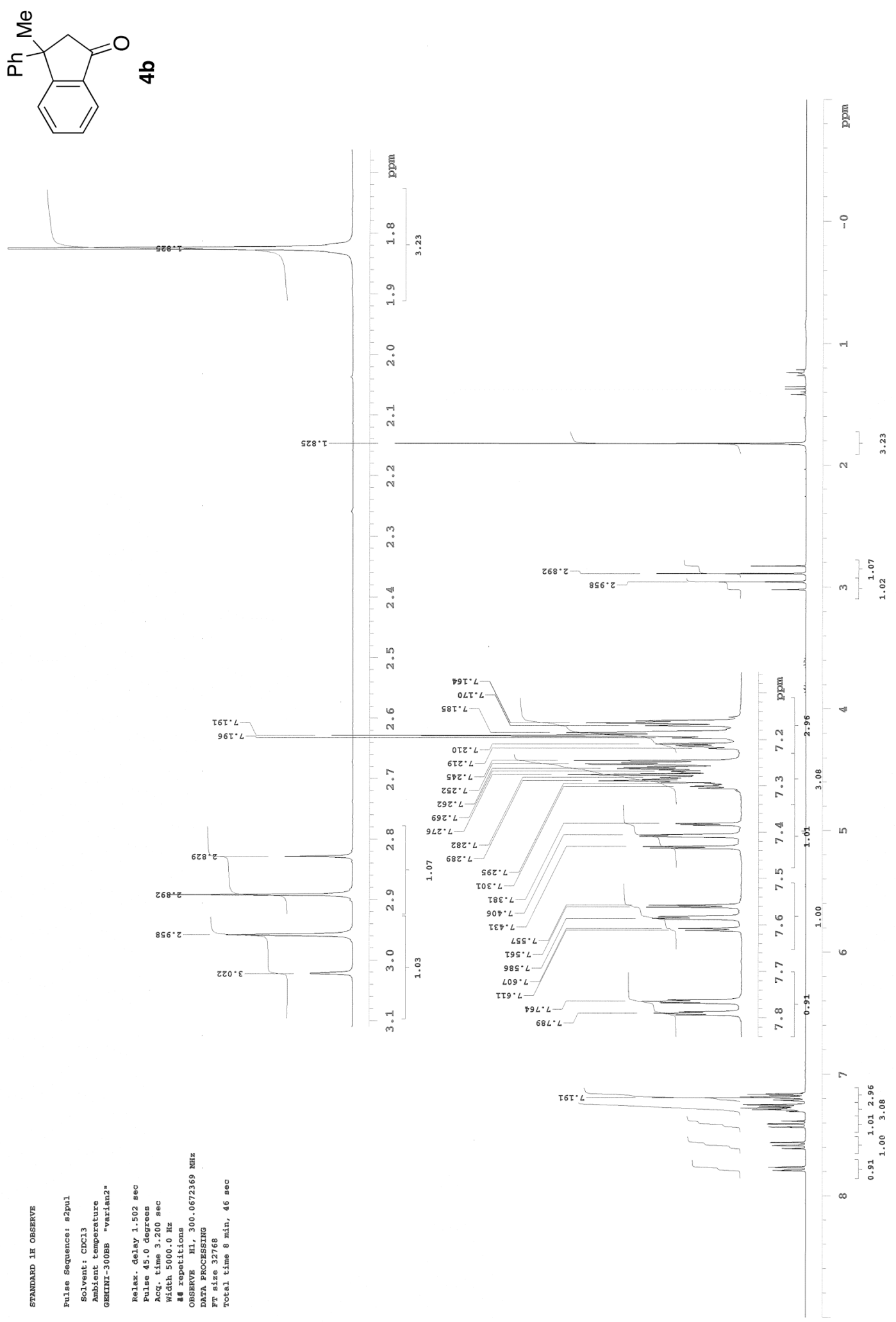



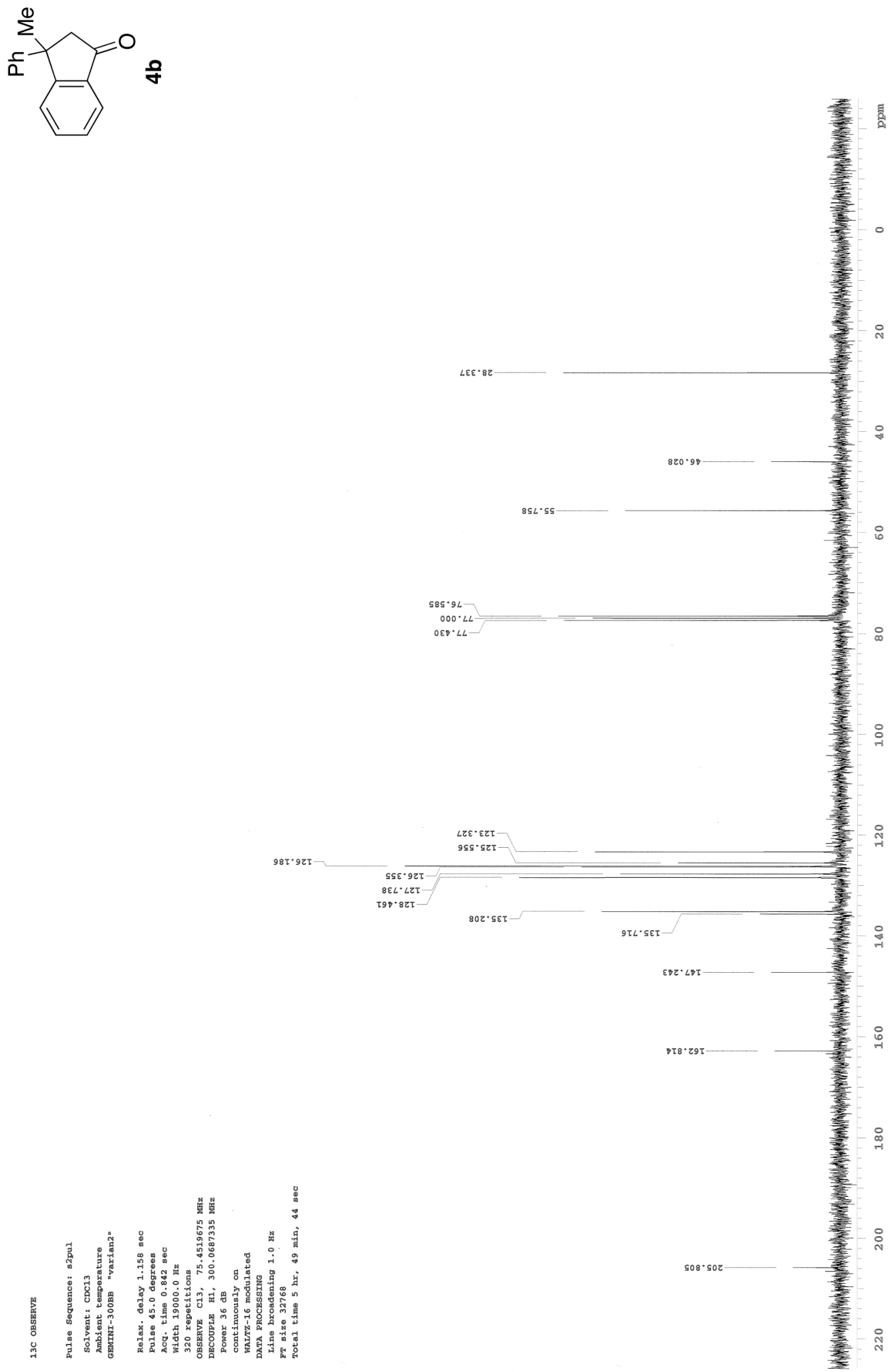

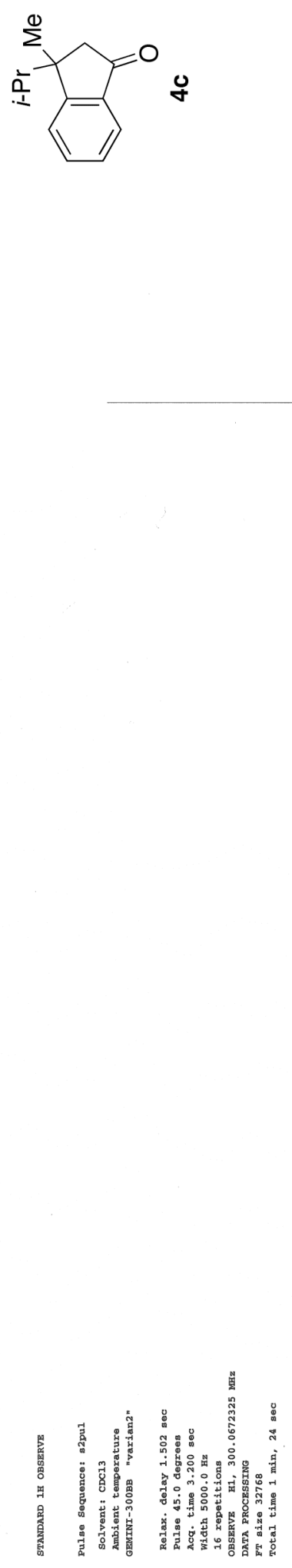

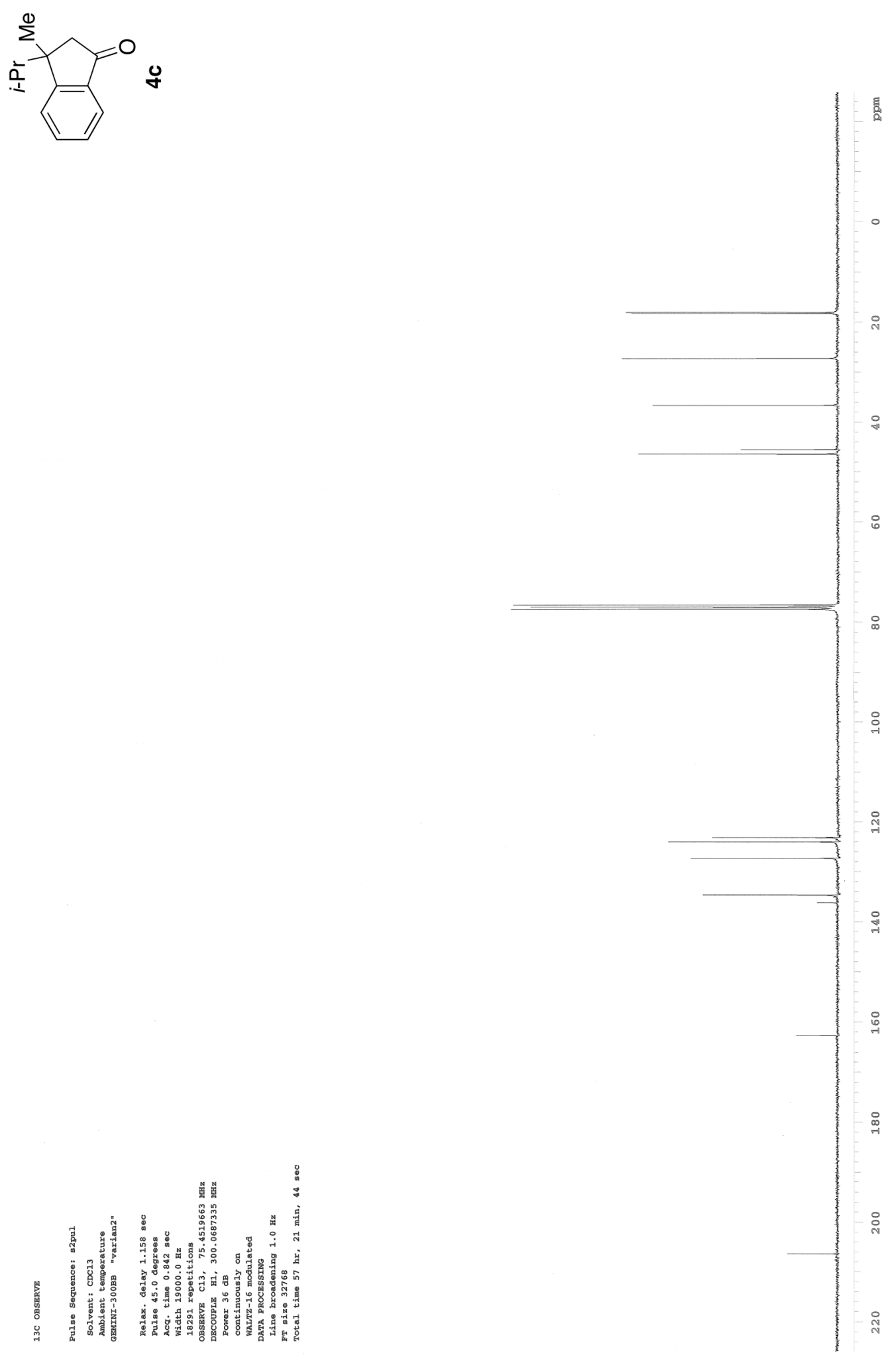


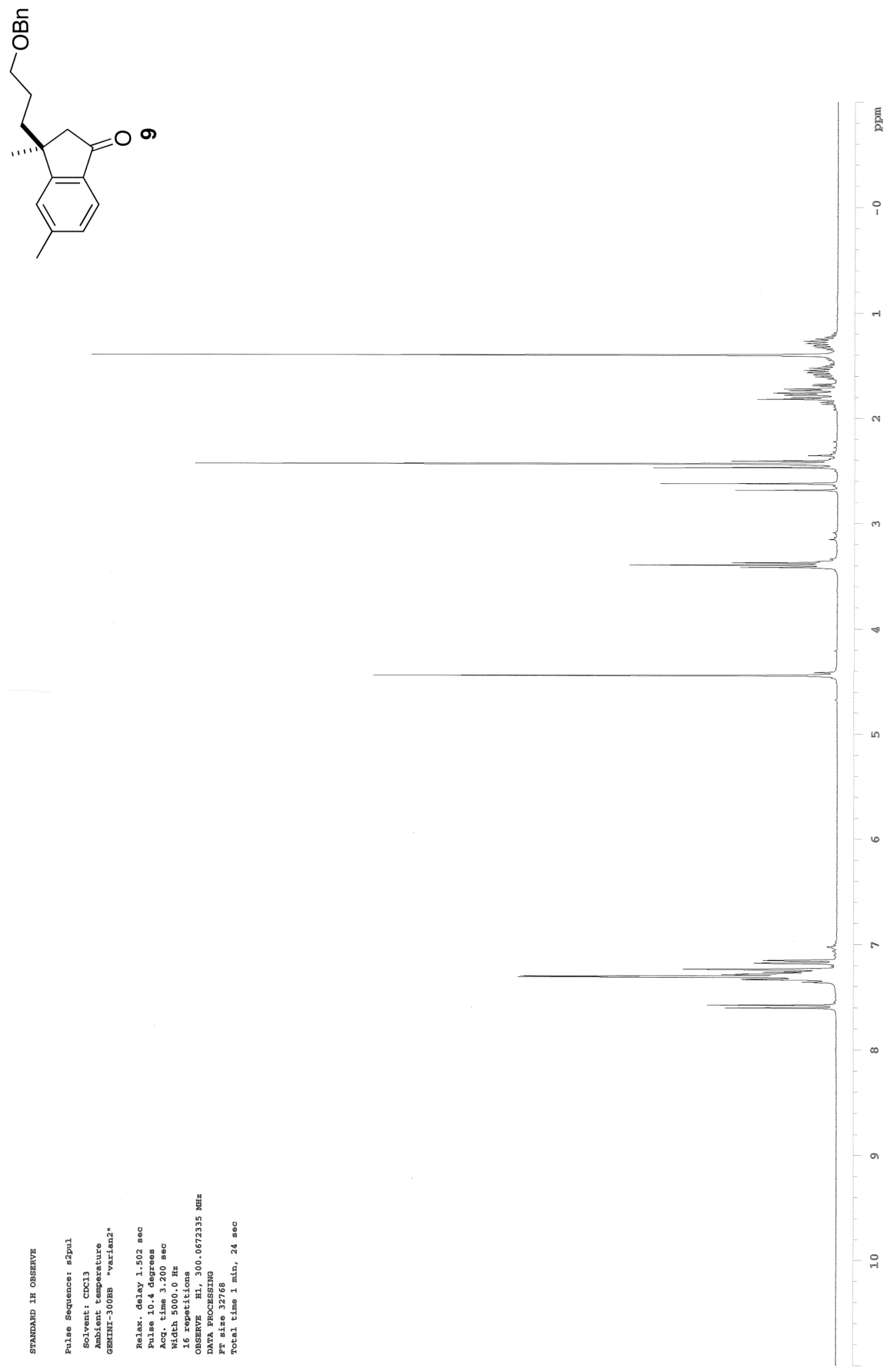



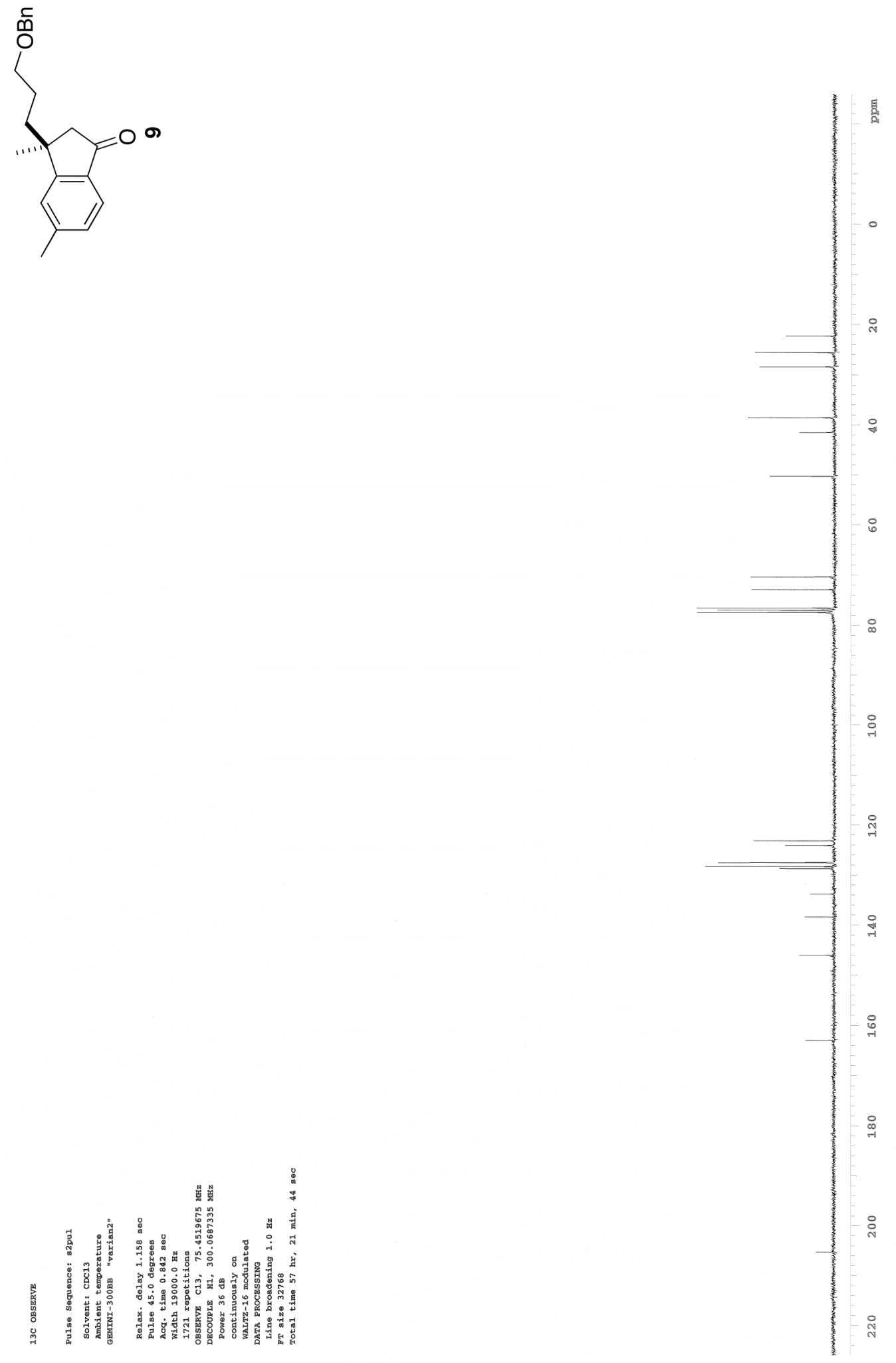


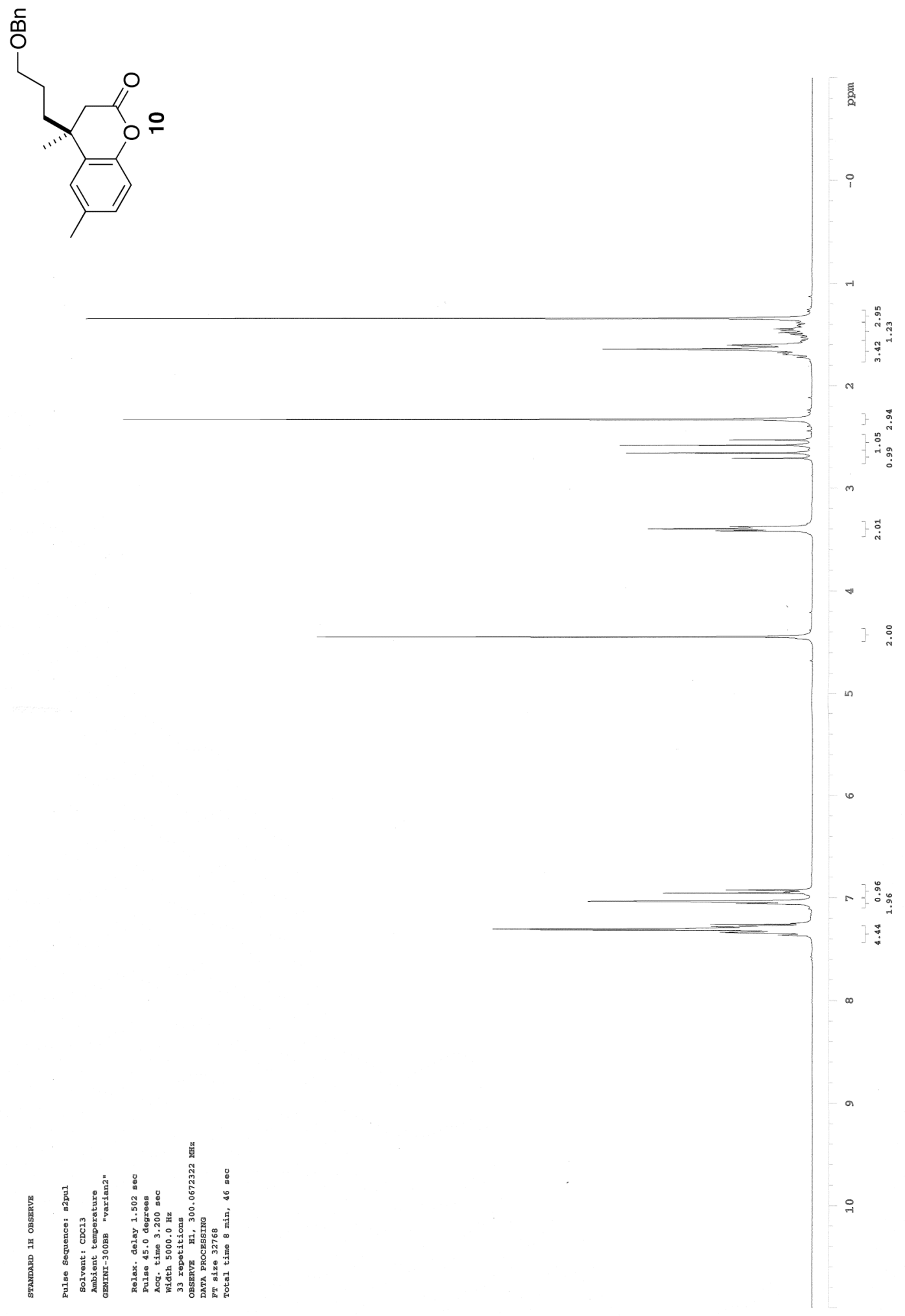



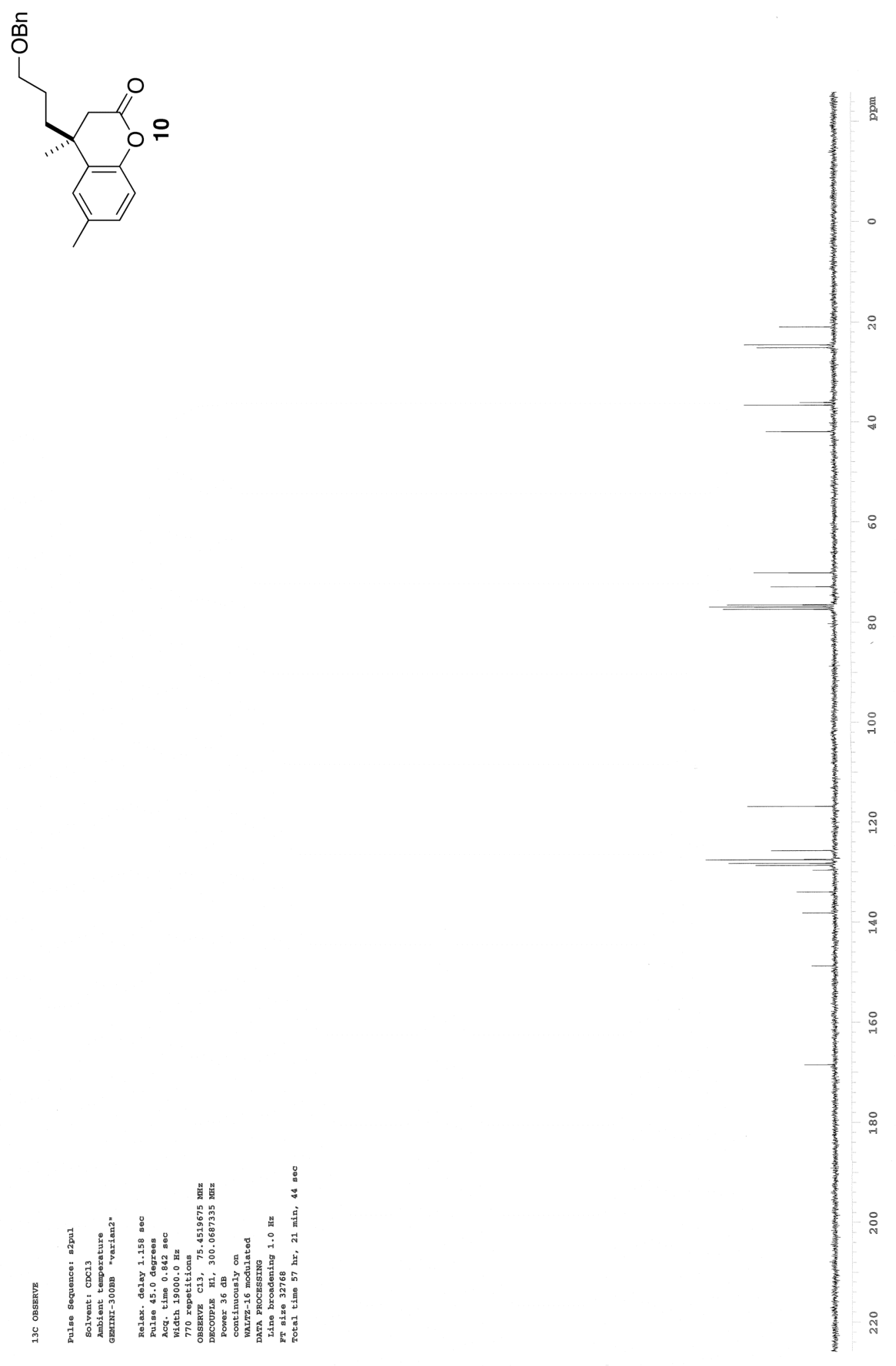


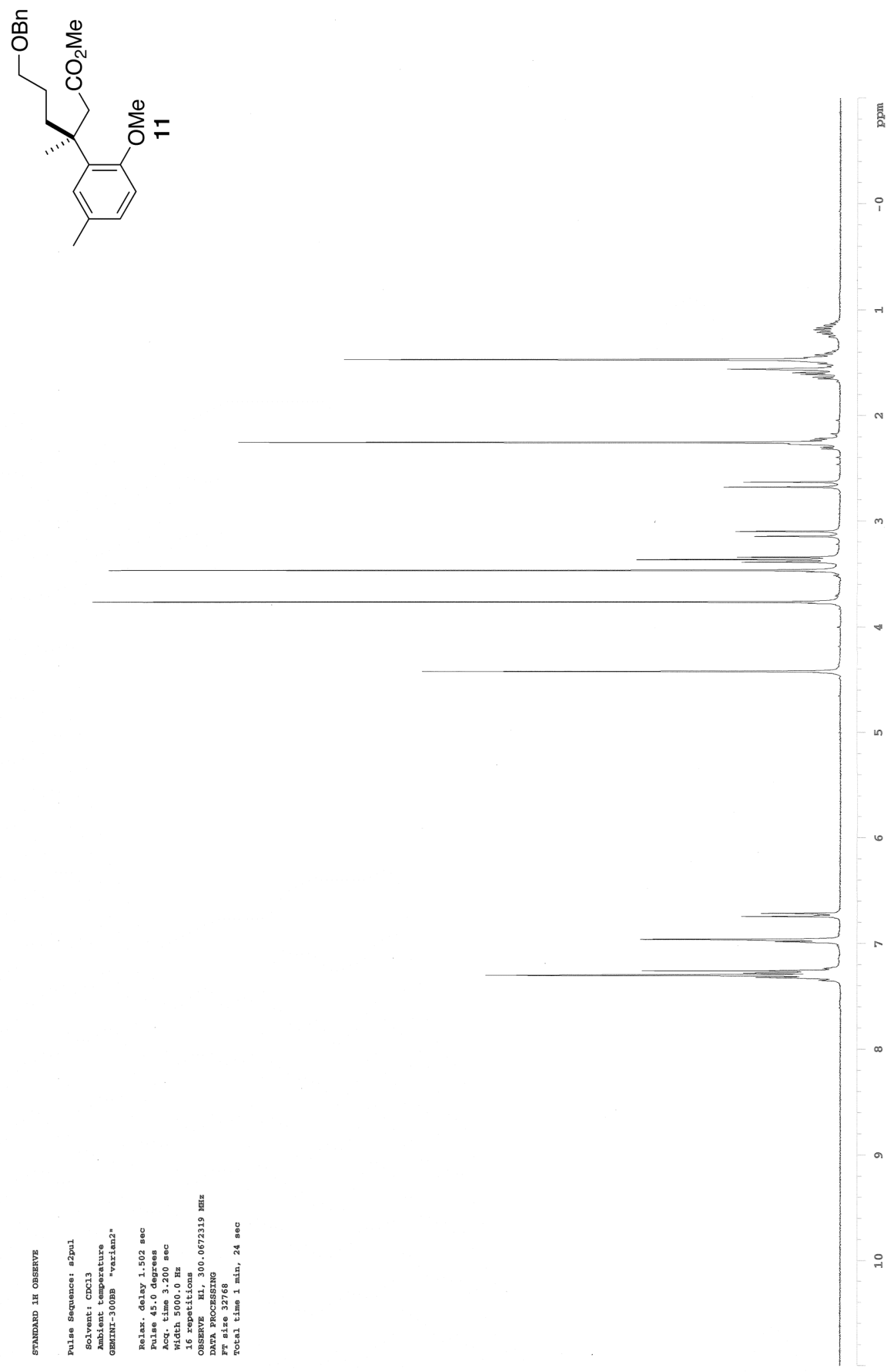



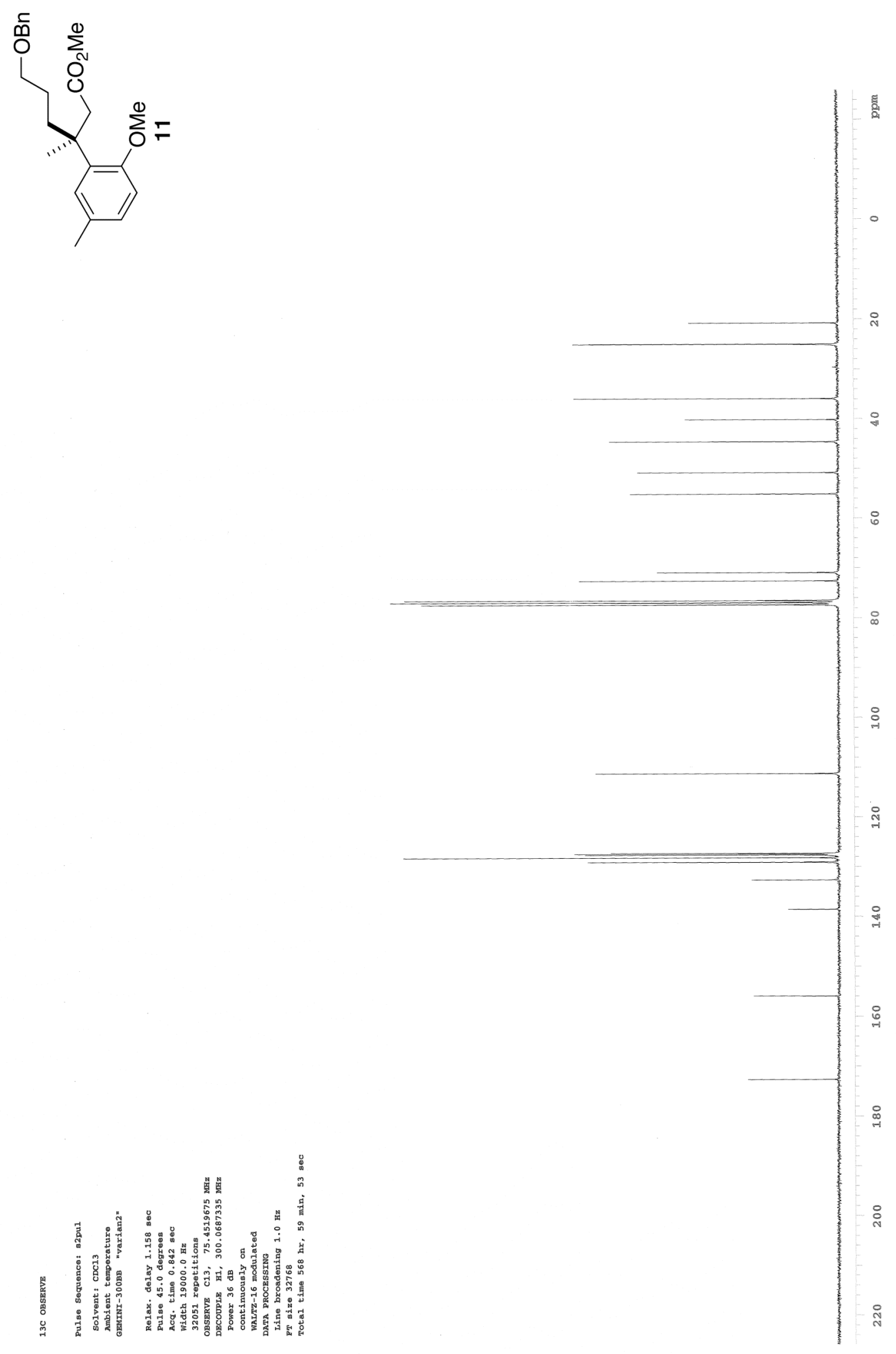


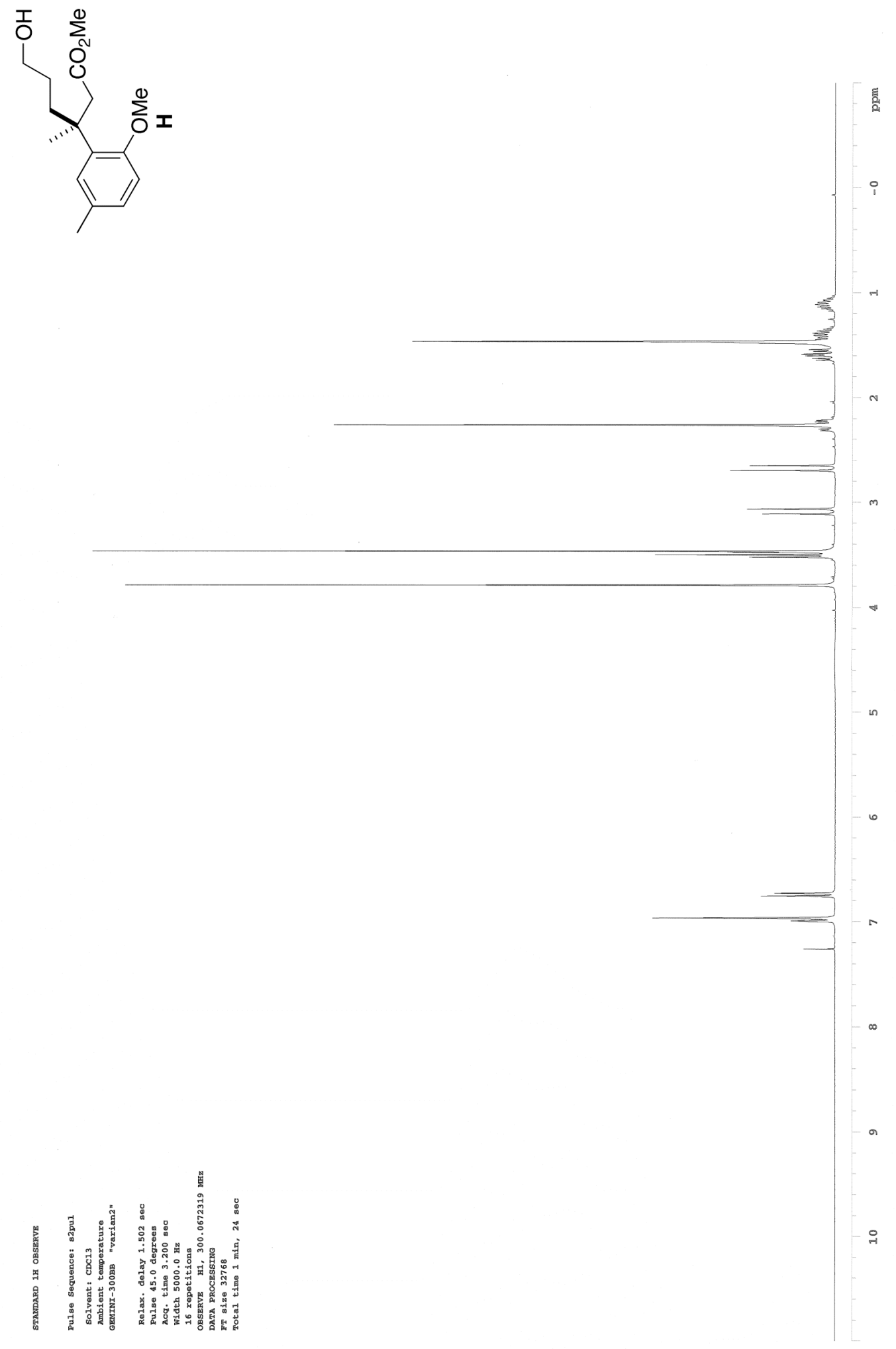




$$
\text { I }
$$

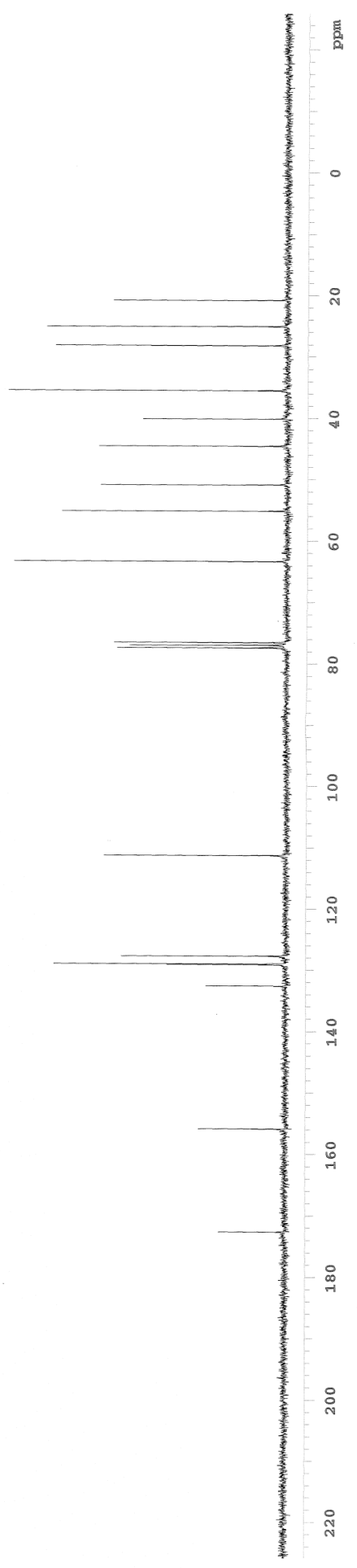



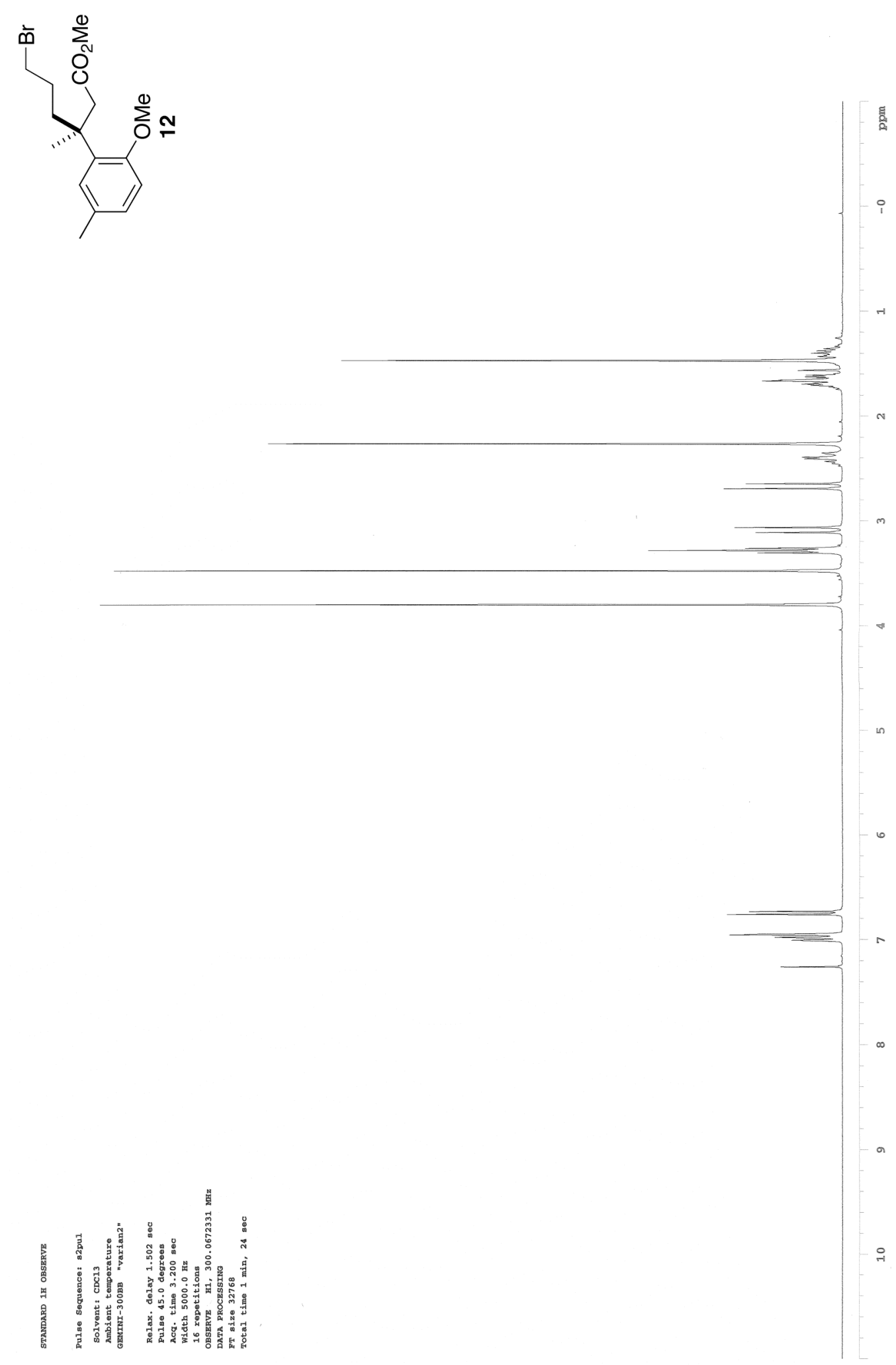

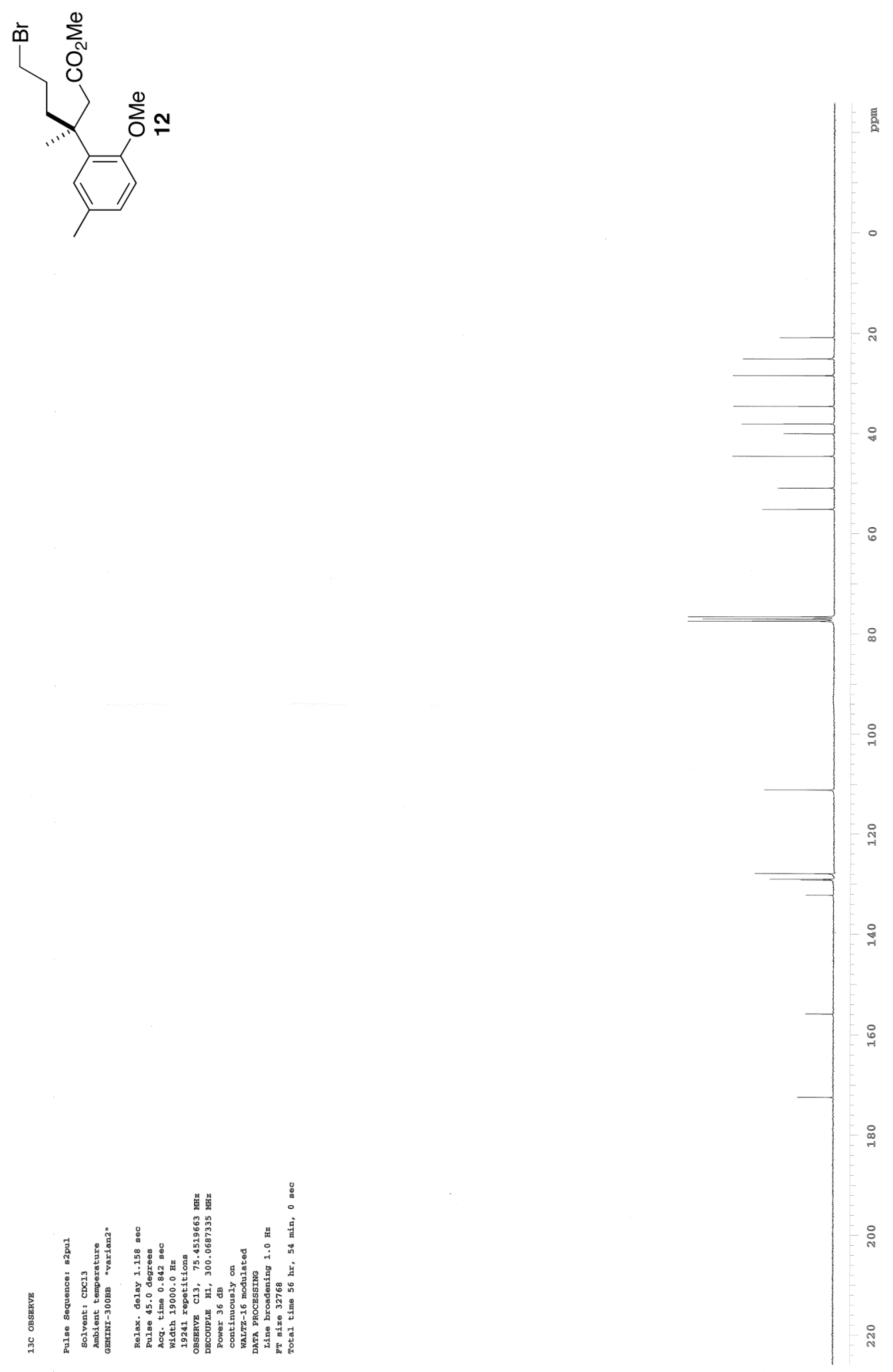


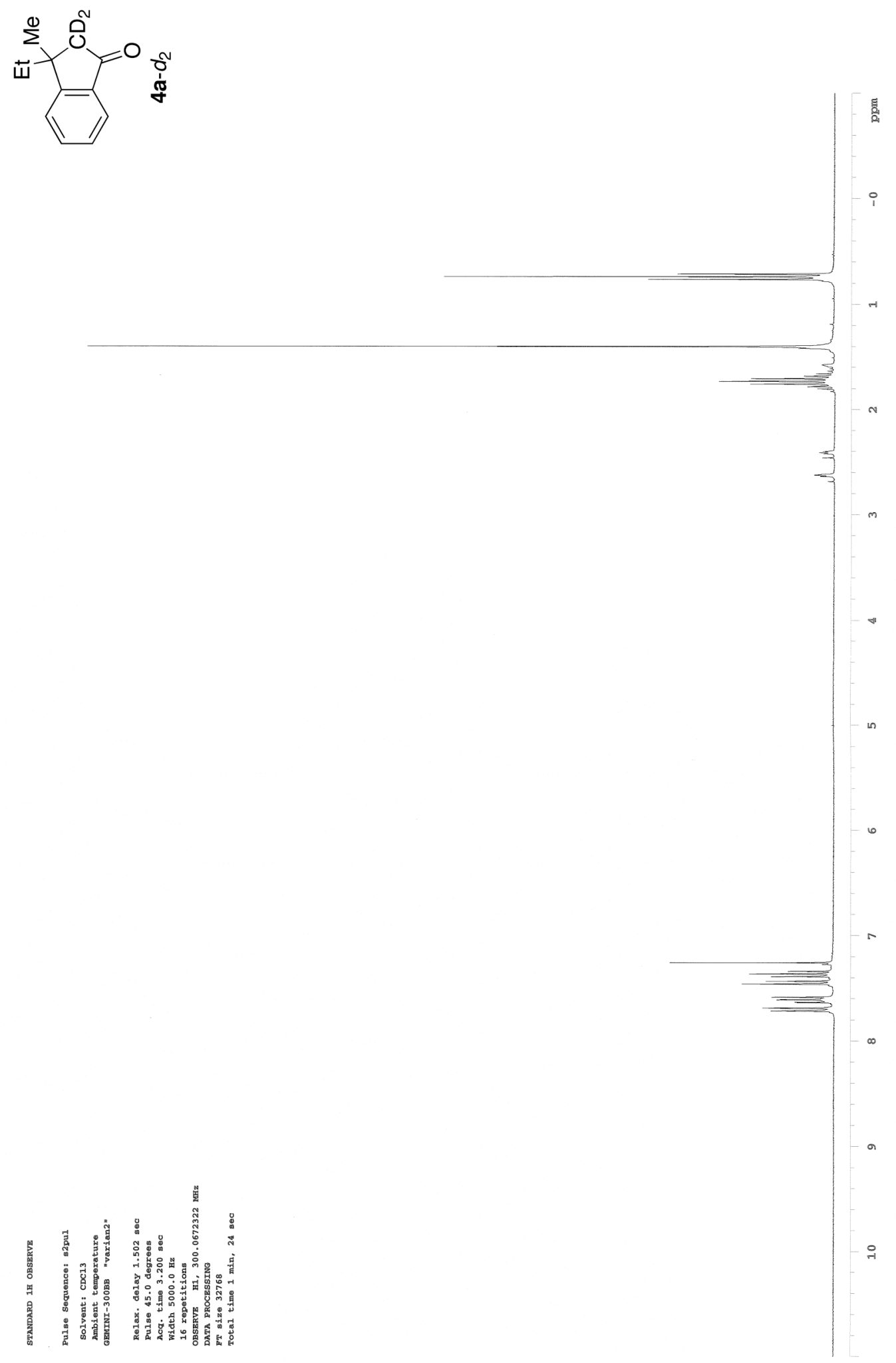




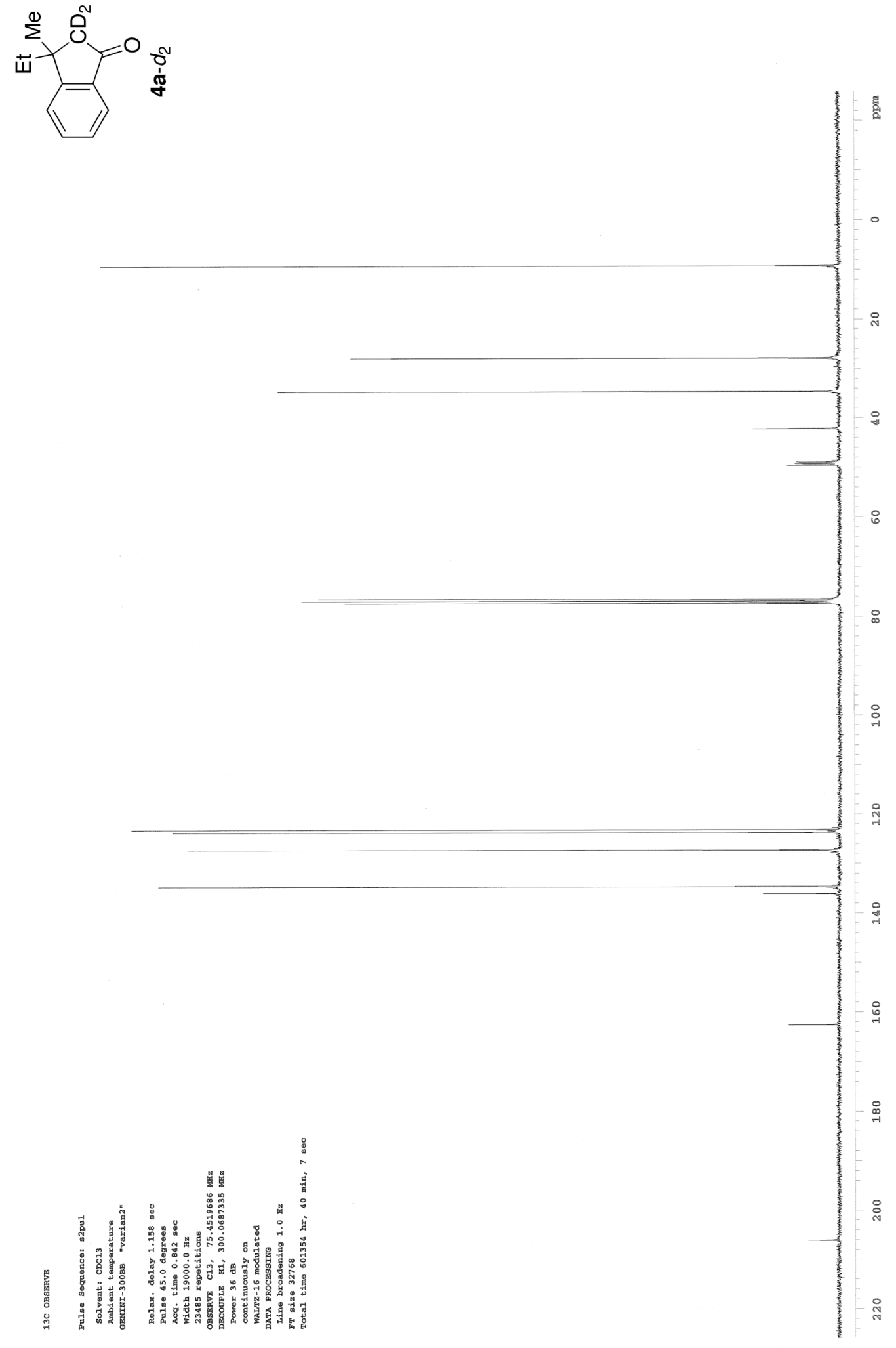




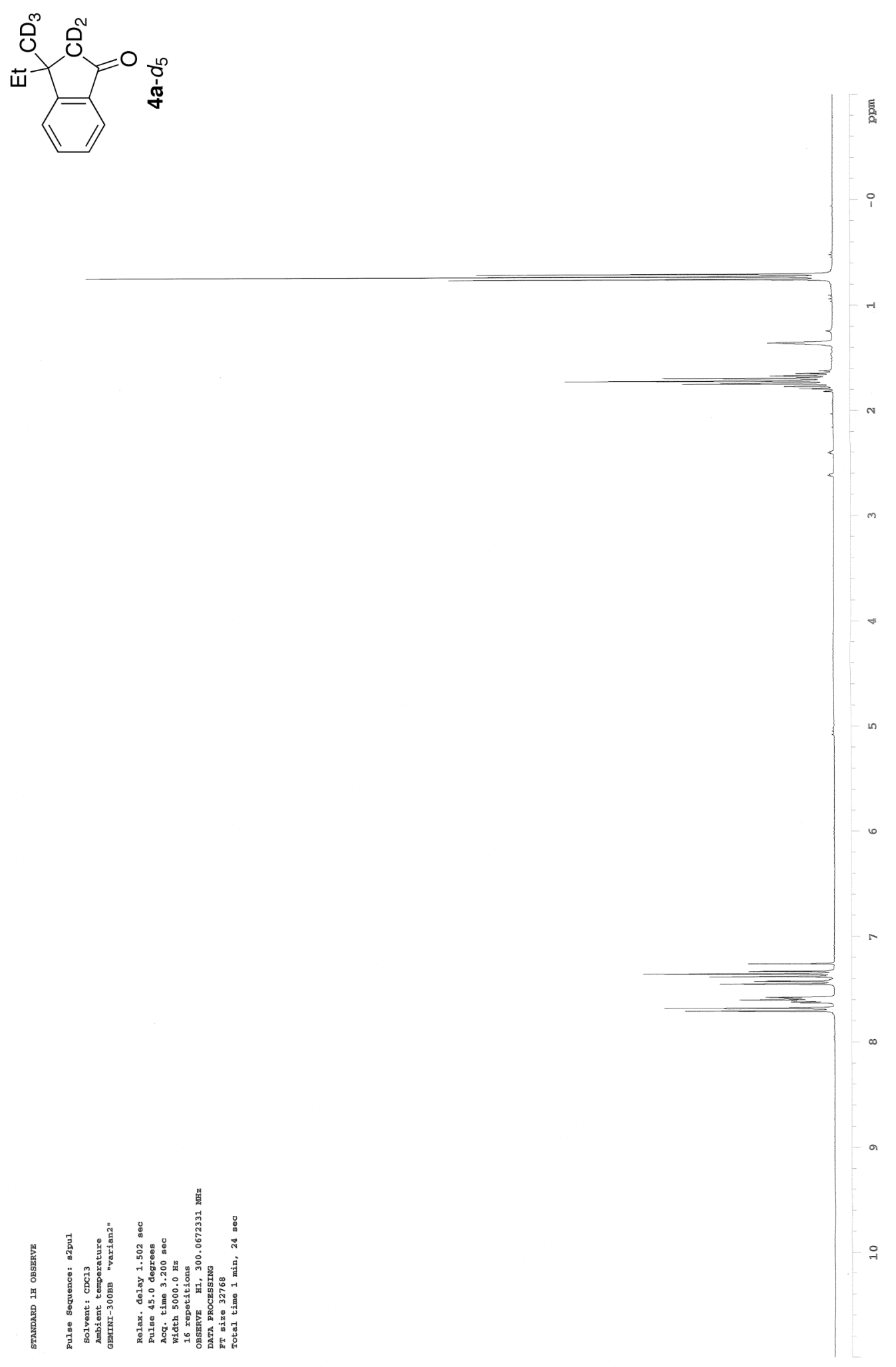



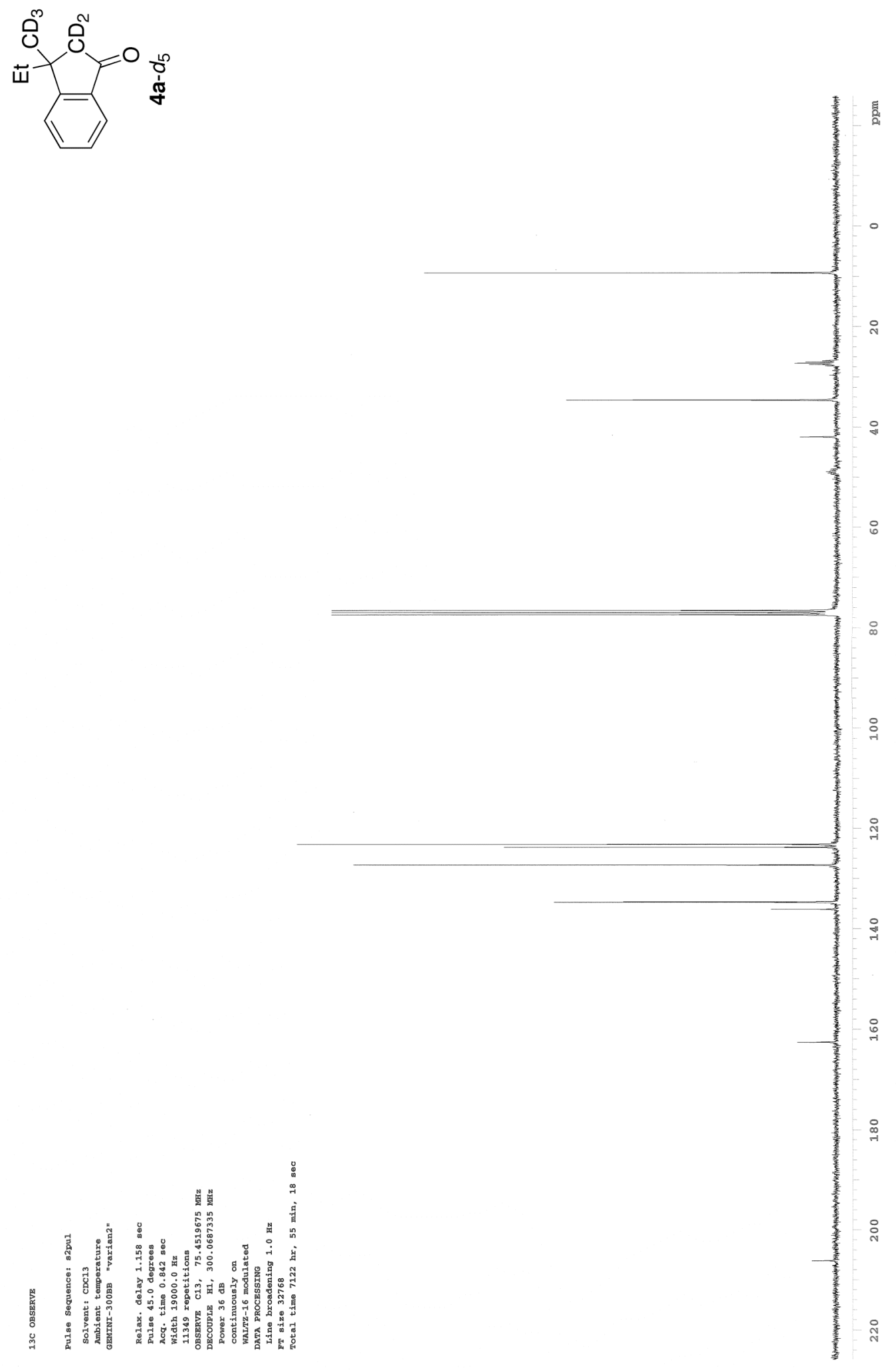